\title{
Incremental Integer Linear Programming Models for Petri Nets Reachability Problems
}

\author{
Thomas Bourdeaud'huy' ${ }^{1}$, Saïd Hanafi² and Pascal Yim ${ }^{1}$ \\ ${ }^{1}$ L.A.G.I.S. Ecole Centrale de Lille \\ ${ }^{2}$ L.A.M.I.H. Université de Valenciennes
}

France

\section{Introduction}

The operational management of complex systems is characterized, in general, by the existence of a huge number of solutions. Decision-making processes must be implemented in order to find the best results. These processes need suitable modeling tools offering true practical resolution perspectives. Among them, Petri nets (PNs) provide a simple graphical model taking into account, in the same formalism, concurrency, parallelism and synchronization. Their graphical and precise nature, their firm mathematical foundation and the aboundance of analysis methods have made them become a classical modeling tool for the study of discrete event systems, ranging from operating systems to logistic ones. However, their interest in the field of problem solving is still badly known.

In this paper, we consider some PN reachability problems. Since PNs can model flows in a natural and efficient way, many operations research problems can be defined using reachability between states, e.g. scheduling (Lee and DiCesare, 1994; Van Der AAlst, 1995), planning (Silva et al., 2000), car-sequencing problems (Briand, 1999). Moreover, research on Petri nets addresses the issue of flexibility: many extensions have been proposed to facilitate the modeling of complex systems, by addition of "color", "time" and "hierarchy" (Jensen, 1992; Wang,1998). For example, it is relatively easy to map scheduling problems onto timed PNs. Their graphical nature reinforce obviously this strength, by allowing a kind of interactivity with the system. At last, a large number of difficult PN analysis problems are equivalent to the reachability problem, or to some of its variants or sub-problems (Keller, 1976). Particularly, model-checking (Latvala, 2001) which represents a key point when dealing with systems analysis is directly linked to an exhaustive traversal of the corresponding PN reachability graph.

Various methods have been suggested to handle the PNs reachability problem. In this paper, we propose to use the mathematical programming paradigm. Some PN analysis problems have already been handled using such techniques (Melzer and Esparza, 1996;Silva et al., 1998; Khomenko and Koutny, 2000), but none has considered the general PNs reachability problem.

The proposed approach is based on an implicit traversal of the Petri net reachability graph, which does not need its construction. This is done by considering a unique sequence of steps growing incrementally to represent exactly the total behavior of the net. We follow here a 
previous work from (Benasser and Yim, 1999) called logical abstraction technique. Their technique was validated on several examples using logical constraint programming techniques. It has shown more effective than other generic solvers and could even compete with heuristics dedicated to particular classes of problems. Our methodology allows to improve this original model using the wide range of tools and adjustments brought by Operational Research techniques. We model the problem as an integer linear program, then we solve it with a branch-and-bound technique (divide and conquer), using the Cplex optimization software.

Moreover, we show how our incremental approach can be extended to Timed Petri nets in order to solve scheduling problems modelled as Timed Petri Nets reachability problems. The model built is as general as possible since we do not make assumptions about the firing policy, contrarywise to other classical approaches dealing with the same issue.

This chapter is organized as follows. In section 2, we formally define the kind of PN considered, their respective reachabilty problems and the ways such problems are dealt with in the litterature. Then, in section 3, we give general considerations about step firings and describe the elements of our incremental approaches. In section 4, we apply our methodology to express reachability problems using a mathematical programming formulation. Finally, as a conclusion, we describe a few promising research directions.

\section{Petri Nets reachability problems}

In this section, we give the terminology of both kinds of the PN we are interested in using linear algebra -- in order to make our formulations more concise -- and define formally their respective reachability problems.

\subsection{Place/transition Petri nets}

\subsubsection{Petri net terminology}

Definition 1 (Place/Transition Petri Net). A Place/Transition Petri net (Murata, 1989) $R=\left(\mathrm{P}, \mathrm{T}, C^{-}, C^{+}\right)$with its initial marking $m$ is a bipartite weighted directed graph where:

- $\quad \mathrm{P}=\left\{p_{1}, \ldots, p_{m}\right\}$ is a finite set of places, with $M=|\mathrm{P}|$. Places are represented as circles and indexed by letter $i$;

- $\mathrm{T}=\left\{t_{1}, \ldots, t_{n}\right\}$ is a finite set of transitions, with $N=|\mathrm{T}|$. Transitions are represented as rectangles and indexed by letter $j$;

- Incidence matrices $C^{-}, C^{+}$and $C \in \mathrm{N}^{\mathrm{P} \times \mathrm{T}}$ (with $C=C^{+}-C^{-}$) define the weighted flow function which associates to each arc $\left(p_{i}, t_{j}\right)$ (from place $p_{i}$ to transition $\left.t_{j}\right)$ or $\left(t_{j}, p_{i}\right)$ (from transition $t_{j}$ to place $p_{i}$ ) its weight $C_{i j}^{-}$or $C_{i j}^{+}$. When there is no arc between place $p_{i}$ and transition $t_{j}$, then we have: $C_{i j}^{-}=C_{i j}^{+}=0$. The $i^{\text {th }}$ row vector and $j^{\text {th }}$ column vector taken from incidence matrices $C^{-}, C^{+}$and $C$ are denoted respectively $C_{i}^{-}, C_{j}^{-}$, $C_{i}^{+}, C_{j}^{+}$and $C_{i}, C_{j}$. We denote respectively by ${ }^{\bullet} p$ and $p^{\bullet}$ the set of predecessors and 
successors of place $p$, and conversely ${ }^{\bullet} t$ and $t^{\bullet}$ are the set of predecessors and successors of transition $t$ (also known as input and output nodes);

- $\quad m: \mathrm{P} \rightarrow \mathrm{N}$ associates to each place $p \in \mathrm{P}$ an integer $m(p)$ called the marking of the place $p$. Markings are represented as full dots called tokens inside places.

Definition 2 (Characteristic Vectors). Let $(R, m)$ be a Petri net with $\mathrm{P}=\left\{p_{1}, p_{2}, \ldots, p_{m}\right\}$ and $\mathrm{T}=\left\{t_{1}, t_{2}, \ldots, t_{n}\right\}$ :

- The canonical vector $\overrightarrow{e_{p_{i}}}$ associated to place $p_{i}$ (resp. $\overrightarrow{e_{t_{j}}}$ associated to transition $t_{j}$ ) is the vector in $\{0,1\}^{N}$ (resp. in $\left.\{0,1\}^{M}\right)$ which takes the value " $1^{\prime \prime}$ in its $i^{\text {th }}$ (resp. $j^{\text {th }}$ ) component and " 0 " elsewhere.

- The marking vector $\vec{m}$ associated to marking $m$ is the column vector $\left(m\left(p_{1}\right), m\left(p_{2}\right)\right.$, $\left.\ldots, m\left(p_{m}\right)\right)^{\dot{\mathrm{u}} \in \mathrm{N}^{M}}$.

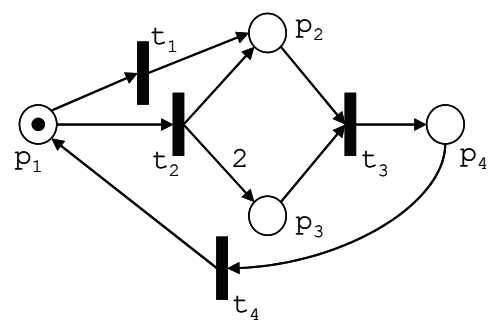

$$
\begin{gathered}
{\left[\begin{array}{llll}
1 & 1 & 0 & 0 \\
0 & 0 & 1 & 0 \\
0 & 0 & 1 & 0 \\
0 & 0 & 0 & 1
\end{array}\right]} \\
C^{-} \\
C^{+}
\end{gathered}
$$

Fig. 1. A Petri Net and its Incidence Matrices

Example 1 (PN). An example of a PN and its incidence matrices is presented in Fig.1. Its initial marking is $m_{0}=(1,0,0,0)^{\dot{\mathrm{u}}}$. We have $\overrightarrow{e_{p_{2}}}=(0,1,0,0,0)^{\dot{\mathrm{u}}}$ and $\overrightarrow{e_{t_{3}}}=(0,0,1,0,0)^{\mathrm{u}}$.

In a PN, the markings of the places represent the state of the corresponding system at a given moment. This state can be modified by the firing of transitions. This behaviour is called the "token game".

Definition 3 (Transition Firings). Let $(R, m)$ be a Petri net. A transition $t_{j}$ is fireable from marking $m$ iff:

$$
\Leftrightarrow \quad \begin{aligned}
\forall p_{i} \in \mathrm{P}, \quad m\left(p_{i}\right) & \geq C_{i j}^{-} \\
\vec{m} & \geq C^{-} \cdot \overrightarrow{e_{t_{j}}}
\end{aligned}
$$

The fireability condition is denoted by $m[t\rangle$. If this condition is satisfied, a new marking $m^{\prime}$ is produced from the marking $m$, such that: 


$$
\begin{aligned}
\forall p_{i} \in \mathrm{P}, \quad m^{\prime}\left(p_{i}\right) & = & m\left(p_{i}\right)-C_{i j}^{-}+C_{i j}^{+} \\
\Leftrightarrow \quad \overrightarrow{m^{\prime}} & = & \vec{m}+C \cdot \overrightarrow{e_{t_{j}}}
\end{aligned}
$$

The firing of a transition $t$ from the marking $m$ to the marking $m^{\prime}$ is denoted by $m[t\rangle m^{\prime}$.

Transition firings modify the marking of the net. It is thus interesting to know if one particular marking can be reached. This problem is known as the "reachability problem" for Petri nets.

\subsubsection{Reachability problem}

Definition 4 (Reachable Marking). A marking $m^{\prime}$ is reachable from a marking $m$ iff there exists a sequence of transitions $\sigma=t_{\sigma_{1}} t_{\sigma_{2}} \ldots t_{\sigma_{k}}$ such that: $m\left[t_{\sigma_{1}}\right\rangle m_{1}\left[t_{\sigma_{2}}\right\rangle m_{2} \ldots\left[t_{\sigma_{K}}\right\rangle m^{\prime}$

We denote by $m[\sigma\rangle m^{\prime}$ that the marking $m^{\prime}$ is reachable from the marking $m$, where $\sigma=t_{\sigma_{1}} t_{\sigma_{2}} \ldots t_{\sigma_{K}}$ is called a firing sequence. The Parikh vector $\vec{\sigma}=\sum_{k=1}^{K} \overrightarrow{e_{t}}$ associated to the firing sequence $\sigma$ is the vector whose $j^{\text {th }}$ component is equal to the number of times the transition $j$ is fired in $\sigma$. It is used to formulate a well known property of Petri Nets.

Proposition 1 (State equation). Let $\left(R, m_{0}\right)$ be a Petri net, $m_{f}$ a marking and $\sigma=t_{\sigma_{1}} t_{\sigma_{2}} \ldots t_{\sigma_{K}}$ a firing sequence. Then we have:

$$
m_{0}[\sigma\rangle m_{f} \Rightarrow \overrightarrow{m_{f}}=\overrightarrow{m_{0}}+C \cdot \vec{\sigma}
$$

Proof. It is obtained using a simple induction over the number of transitions fired in the sequence.

The equation Error! Reference source not found. is called the fundamental (or state) equation of Petri nets. This equation has been widely studied in PN reachability analysis, but it only leads to semi-decision algorithms due to the existence of spurious solutions (Silva et al., 1992). Indeed, in that case, the reverse implication does not hold: the Parikh vector of a firing sequence is always solution to the state equation, but the reverse is not true. Some techniques (Colom and Silva, 1989b) have been proposed to improve the strength of this characterization, but they are still insufficient.

Definition 5 (Reachability Problem). Let $\left(R, m_{0}\right)$ be a Petri net and $m_{f}$ a marking. The set of all markings reachable from $m_{0}$ is denoted by $\mathrm{R}\left(R, m_{0}\right)$; the set of all possible firing sequences (within which each transition is fireable from the corresponding marking) is denoted by $\mathrm{F}\left(R, m_{0}\right)$. The problem of finding whether $m_{f} \in \mathrm{R}\left(R, m_{0}\right)$ or not is known as the reachability problem for Petri nets.

It has been shown that the reachability problem is decidable (Kosaraju, 1982). However it is EXP-TIME and EXP-SPACE hard in the general case (Lipton, 1976). Of course, practical 
applications need not only to know if a marking is reachable, but also what are the corresponding firing sequences leading to this marking. To solve this problem, one needs to find a firing sequence $\sigma \in \mathrm{F}\left(R, m_{0}\right)$ such that $m_{0}[\sigma\rangle m_{f}$. A "naive" approach consists in exploring the reachability graph exhaustively. This graph corresponds to the usual formal representation of the behavior of the net.

Definition 6 (Reachability Graph). The reachability graph of a Petri net $\left(R, m_{0}\right)$, denoted by $\mathrm{G}\left(R, m_{0}\right)$, is defined by:

A set of nodes $\mathrm{R}\left(R, m_{0}\right)$ which represents the reachable markings;

A set of arcs, where an arc $\left(m, m^{\prime}\right)$ labelled $t$ connects nodes $m$ and $m^{\prime}$ iff $m[t\rangle m^{\prime}$.

Example 2 (Reachability Graph). Fig.2. presents a part of the reachability graph for the Petri net of Fig.1.

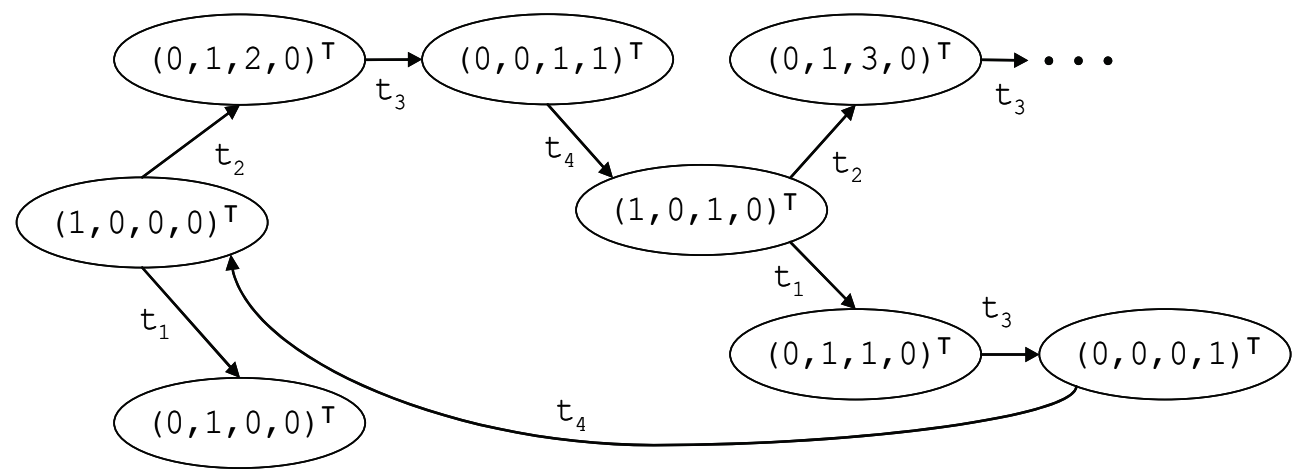

Fig. 2. Reachability graph for the PN of Fig. 1

For a given initial marking $m_{0}$, the reachability graph $\mathrm{G}\left(R, m_{0}\right)$ and the corresponding reachability set $\mathrm{R}\left(R, m_{0}\right)$ may be of infinite size. For instance, the set of markings reachable from $m_{0}$ for the net of Fig. 1 is infinite.

Practically, it is not possible to explore the reachability graph exhaustively due to the well known problem of combinatorial explosion: the size of the state-space (i.e. the size of the reachability set) may grow exponentially with the size of a system configuration (i.e. the number of nodes of the Petri net). Many methods have been studied to limit this explosion. Let us mention the three main families.

First ones aims at managing the combinatorial explosion without modifying the studied reachability graph. Classical approaches are graph compressions, particularly bdd encoding (Gunnarsson, 1998) and forward checking (Fernandez et al., 1992). Both uses depth first traversal of the reachability graph.

- Other techniques construct a reduced reachability graph associated to the original, based on some properties to preserve: symmetries (Huber et al., 1985), reductions (Berthelot, 1986) and partial order (covering step graphs (Vernadat et al., 1996), stubborn sets 
(Valmari, 1991)) are the main approaches. The logical abstraction technique (Benasser and Yim, 1999) belongs also to this category.

- $\quad$ Last ones are based on the PN state equation (cf. Proposition 1): we can distinguish parametrized analysis (Lindqvist, 1993) and algebraic methods (Lautenbach, 1987).

Many extensions have been proposed to improve the modelling power of Petri nets. Among them, several extended Petri nets with "time" have been proposed by assigning punctual firing times (leading to "Timed $P N^{\prime}$ ) or time intervals ("Time $P N^{\prime \prime}$ ) to the components of Petri nets (transitions, places, arcs or even tokens). To deal with firing times, two main methods for modeling timing are used: either the timings are associated with the places (the PN is said to be P-timed) (Sifakis, 1975), or the timings are associated with the transitions (the PN is said to be T-timed) (Ramchandani, 1974). Depending on the system to be modeled, one of the models (P-timed or T-timed) may be easier to use than the other one. However, Sifakis has shown that the two models are equivalent. In the context of scheduling problems, (Hillion and Proth, 1989) and (Van Der Aalst, 1995) propose to use T-timed Petri nets, hereafter called simply Timed PN. We describe this model in the following section.

\subsection{Timed Petri nets}

Timed Petri nets have been introduced by (Ramchandani, 1974). The following presentation has been adapted from (Chrétienne, 1984). We start by giving an informal introduction on Timed Petri nets.

\subsubsection{Informal presentation}

Timed Petri nets correspond to Places/Transitions Petri nets where a duration $d(t) \in \mathrm{N}^{*}$ is associated to each transition $t$. A Timed Petri net has the same representation as PN, to which is added a labelling on transitions. An example of Timed Petri net is given in Fig. 3. We have: $d\left(t_{1}\right)=3, d\left(t_{2}\right)=4, d\left(t_{3}\right)=5, d\left(t_{4}\right)=2$.

The firing durations associated to transitions modify the marking validity conditions. As soon as durations are associated to transitions, the Petri net acts as if tokens "disappeared" at the time the transition is fired, and then "reappeared" after a delay corresponding to the duration of the fired transition. Thus, the marking of a Timed Petri net evolves with the occurences of an external timer. For instance, let's consider the Timed Petri net of Fig. 3. At date 1 , the transition $t_{1}$ (duration: 3 t.u.) is fired. Then the transition $t_{4}$ (duration: 2 t.u.) is fired at date 5 . The evolution of marking with time is given in Fig. 3. Note that one could have fired transition $t_{4}$ at date 4 , since the resource $r_{1}$ had been released at the end of the firing of transition $t_{1}$. However, the same transition was not fireable at date 3 , since the firing of $t_{1}$ was not finished.

The firing and ending dates of transitions play a fundamental role in the behaviour of the Timed Petri net. It is thus necessary to adapt the firing equations according to these firing dates. In order to respect the underlying semantic of PN, a timed firing sequence is said to be feasible if and only if, at any time, the transient marking reached is made of non negative components. 


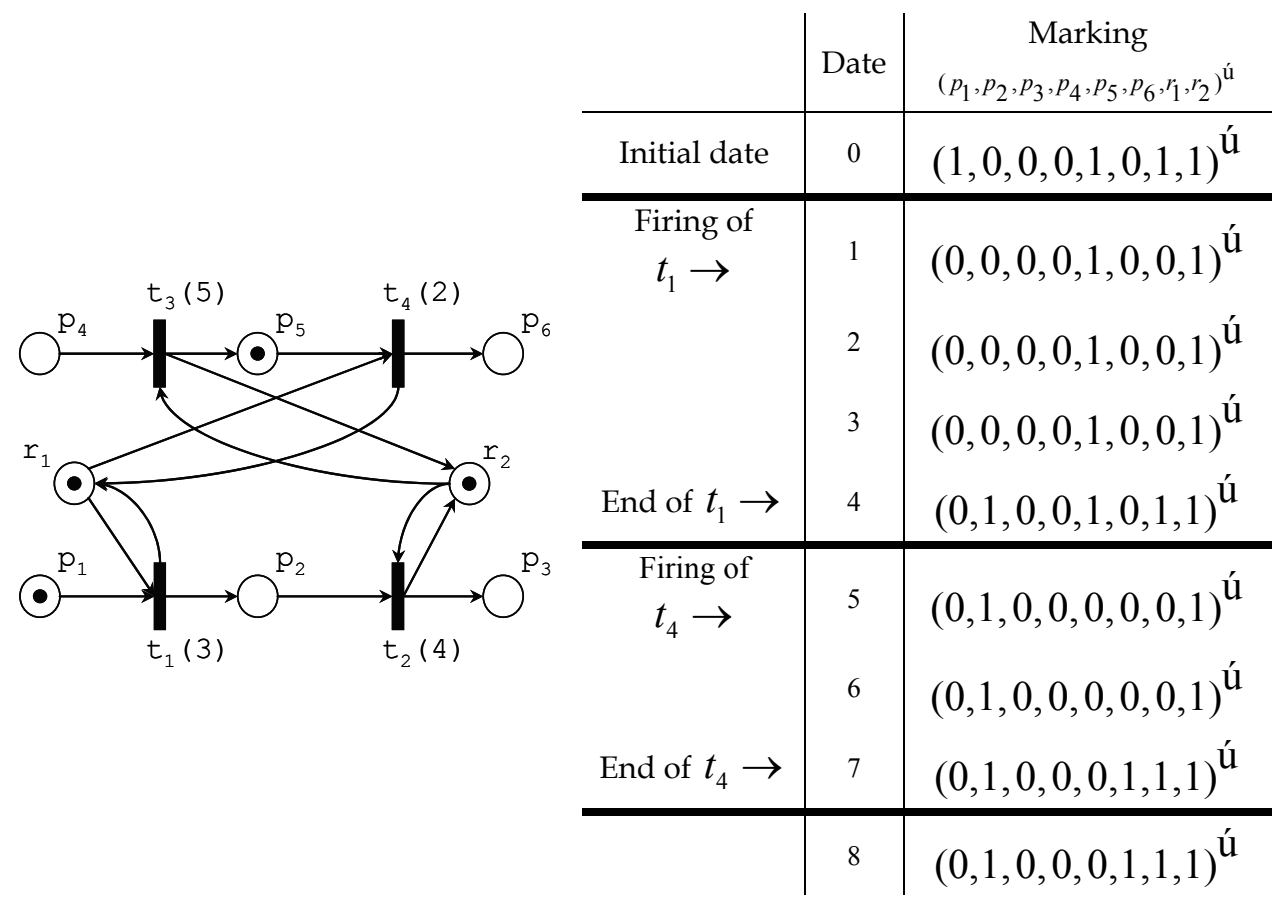

Fig. 3. Example of a Timed Petri Net and a Timed Firing Sequence

\subsubsection{Timed Petri nets terminology}

Definition 7 (TPN -- Timed Petri Net). A Timed Petri net (Ramchandani, 1974) is defined by a pair $(R, d)$ where $R$ is a Place/Transition Petri Net and $d: \mathrm{T} \rightarrow \mathrm{N}^{*}$ is a mapping associating a duration to each transition of the net. The vector $\vec{d}=\sum_{t \in \mathrm{T}} d(t) \cdot \overrightarrow{e_{t}}$ is called the duration vector of the Timed Petri net.

Note that one could more generally consider rational valued durations. Nevertheless, after having them reduced to the same denominator, and by reasoning over numerators, it is the same as if durations were integer valued. In addition, to simplify the study, we restrict ourselves to Timed Petri nets without immediate transitions (i.e. $\forall t \in \mathrm{T}, d(t)>0$ ), which is not so restrictive in real world practice and corresponds well to scheduling problems we are concerned with.

The transition firing semantics in TPN forbids reentrance. In other words, it is not possible to fire again a transition that has not yet finished to be fired. Again, this semantics is well fitted to scheduling problems, where transitions are associated to operations on machines. Thus, one can associate a unique residual duration to each transition without any possible confusion between several concurrent transitions activations. The residual duration vector is associated to the marking of a TPN to define its full state.

Definition 8 (TPN State). Let $(R, d)$ be a TPN. Its state $e=\left(\overrightarrow{E_{m}}, \overrightarrow{E_{r}}\right)$ is given by: 
- Its classical marking vector $\overrightarrow{E_{m}} \in \mathrm{N}^{M}$, associating to each place its number of tokens;

- A residual durations vector $\overrightarrow{E_{r}} \in \mathrm{N}^{N}$, associating to each active transition its remaining duration, and zero if the transition is not active.

The set of all states of a TPN is denoted by $\mathrm{S}(R, d)$. The fundamental concept that governs Timed Petri net behavior is the controlled execution, which associates to each transition the sequence of its successive firing dates.

Definition 9 (CE - Controlled Execution) Let $(R, d)$ be a TPN and $t \in \mathrm{T}$ a transition. A firing sequence for the timed transition $t:\left(u_{k}^{t}\right)=u_{1}^{t}, \ldots, u_{K_{t}}^{t} \in \mathrm{N}$ is an increasing sequence of firing dates, such that:

$$
\forall k \in \llbracket 1, K_{t}-1 \rrbracket, u_{k}^{t}+d(t) \leq u_{k+1}^{t}
$$

A controlled execution is a family $\left(u_{k}^{t}\right)_{t \in \mathrm{T}, k \in \llbracket 1, K_{t} \rrbracket}$ of firing sequences for all transitions of the TPN. Note that in the previous definition, equation (4) is used to forbid reentrance. For any transition $t, k_{t}$ and $u_{K_{t}}^{t}$ may be infinite. Hereafter, we only consider finite CEs. We denote by $v_{\max }$ the ending date of the last firing in the CE: $v_{\max }=\max _{t \in \mathrm{T}}\left(u_{K_{t}}^{t}+d(t)\right)$. After $v_{\text {max }}$, the state of the TPN under the considered CE will never change and we have: $\overrightarrow{E_{r}\left(v_{\max }\right)}=\overrightarrow{0_{N}}$.

The formal expression of a CE is used to define several characteristic vectors allowing to verify the feasability of a $\mathrm{CE}$. We assume that no transition is active at the initial state to simplify the formulation.

Definition 10 (Characteristic Vectors of Controlled Executions) Let $(R, d)$ be a TPN with its initial state $e_{0}=\left(\overrightarrow{E_{m_{0}}}, \overrightarrow{0_{N}}\right)$ given at initial date 0 and $\left(u_{k}^{t}\right)_{t \in \mathrm{T}, k \in \llbracket 1, K_{t} \rrbracket}$ a controlled execution. Let $v \in \llbracket 0, v_{\max } \rrbracket$. We define three characteristic vectors associated to $\left(u_{k}^{t}\right)$ in the following way:

$\overrightarrow{N(v)} \in \mathrm{N}^{N}$ is the vector corresponding to the number of firings that started within the interval $[0, v]$, defined by $\left.\overrightarrow{N(v)}\right|_{t}=\operatorname{card}\left(\left\{u_{k, k \in \llbracket 1, K_{t} \rrbracket}^{t} \mid u_{k}^{t} \leq v\right\}\right)$;

- $\overrightarrow{D(v)} \in \mathrm{N}^{N}$ is the vector corresponding to the number of firings that started within the interval $\left[0, v\left[\right.\right.$, defined by $\left.\overrightarrow{D(v)}\right|_{t}=\operatorname{card}\left(\left\{u_{k, k \in \llbracket 1, K_{t} \rrbracket}^{t} \mid u_{k}^{t}<v\right\}\right)$; 
- $\overrightarrow{F(v)} \in \mathrm{N}^{N}$ is the vector corresponding to the number of firings that ended within the interval $[0, v]$, defined by $\left.\overrightarrow{F(v)}\right|_{t}=\operatorname{card}\left(\left\{u_{k, k \in \llbracket 1, K_{t} \rrbracket}^{t} \mid u_{k}^{t}+d(t) \leq v\right\}\right)$.

We have introduced above the definitions of state and controlled execution of a TPN. We define below how the state of a TPN is modified under a CE.

Definition 11 (Instantaneous State of a TPN under a Controlled Execution) Let $(R, d)$ be a TPN with its initial state $e_{0}=\left(\overrightarrow{E_{m_{0}}}, \overrightarrow{0_{N}}\right)$ given at date 0 and $\left(u_{k}^{t}\right)_{t \in \mathrm{T}, k \in \llbracket 1, K_{t} \rrbracket}$ controlled execution. Let $v \in \llbracket 0, v_{\max } \rrbracket$. The instantaneous state $e_{v}=\left(\overrightarrow{E_{m}(v)}, \overrightarrow{E_{r}(v)}\right)$ at date $v$ is given by:

$$
\begin{gathered}
\overrightarrow{E_{m}(v)}=\overrightarrow{E_{m_{0}}}+C^{+} \cdot \overrightarrow{F(v)}-C^{-} \cdot \overrightarrow{N(v)} \\
\forall t \in \mathrm{T},\left.\overrightarrow{E_{r}(v)}\right|_{t}= \begin{cases}u_{k}^{t}+d(t)-v & \text { if } \exists k \in \llbracket 1, K_{t} \rrbracket s . t . v \in \llbracket u_{k}^{t}, u_{k}^{t}+d(t) \llbracket \\
0 & \text { otherwise }\end{cases}
\end{gathered}
$$

Informally, in the previous definition, the quantity $C^{+} \cdot \overrightarrow{F(v)}$ corresponds to the tokens produced by the firings of transitions that ended strictly before the date $v$. Those tokens can be used to fire transitions at date $v$. The quantity $C^{-} \cdot \overrightarrow{N(v)}$ corresponds to the tokens used by the firings of transitions that started until the date $v$. Thus, the quantity $\overrightarrow{E_{m}(v)}$ corresponds exactly to the tokens remaining in the TPN at date $v$. The residual durations vector $\overrightarrow{E_{r}(v)}$ denotes the exact remaining time of transitions that are active at date $v$. Obviously, there can only be one $k \in \llbracket 1, K_{t} \rrbracket s . t . v \in \llbracket u_{k}^{t}, u_{k}^{t}+d(t) \llbracket$ from equation (4). Note that (Chretienne, 1984) defines also the quantity $\overrightarrow{m^{-}(v)}=\overrightarrow{E_{m}(0)}+C^{+} \cdot \overrightarrow{F(v)}-C^{-} \cdot \overrightarrow{D(v)}$. This quantity does not consider the tokens used by the firings of transitions that occur exactly at date $v$. Thus, it can be used to formulate the fireability condition for a transition in a TPN, independently from possible concurrent activations: under a controlled execution, a transition is fireable at date $v$ iff $\overrightarrow{m^{-}(v)} \geq C^{-} \cdot \overrightarrow{e_{t}}$.

Obviously, like for Place/Transitions PNs, even if each transition is independently fireable at every date, the full CE is not necessarily valid as a whole since token may be used by several transitions at the same time. Thus, an improved condition for a CE to be feasible is given below. 
Definition 12 (Feasible Controlled Execution). Let $(R, d)$ be a TPN with its initial state $e_{0}=\left(\overrightarrow{E_{m_{0}}}, \overrightarrow{0_{N}}\right)$ given at date 0 and $\left(u_{k}^{t}\right)_{t \in \mathrm{T}, k \in \llbracket 1, K_{t} \rrbracket}$ a controlled execution. This controlled execution is said to be feasible iff:

$$
\forall v \in \llbracket 0, v_{\max } \rrbracket, \overrightarrow{E_{m}(v)} \geq \overrightarrow{0_{M}}
$$

The previous condition means that there must be enough tokens so that transitions may fire simultaneously.

\subsubsection{Timed Petri Net Reachability Problem}

Using the previous notations, the Timed Petri nets reachability problem consists in searching for a feasible $C E$ allowing to reach a given final state from the initial state.

Definition 13 (Timed PN Reachability Problem). Let $(R, d)$ be a TPN with its initial state $e_{0}=\left(\overrightarrow{E_{m_{0}}}, \overrightarrow{0_{N}}\right)$ given at date 0 . Let $e_{f}=\left(\overrightarrow{E_{m_{f}}}, \overrightarrow{0_{N}}\right)$ be a target state. The reachability problem for Timed Petri nets consists in finding a CE $\left(u_{k}^{t}\right)_{t \in \mathrm{T}, k \in \llbracket 1, K_{t} \rrbracket}$ such that $e_{v_{\max }}=\left(\overrightarrow{E_{m}\left(v_{\max }\right)}, \overrightarrow{E_{r}\left(v_{\max }\right)}\right)=e_{f}$.

As said before, it is quite simple to see a parallelism between a scheduling problem and a Timed Petri net reachability problem. Indeed, let's consider for instance the Timed Petri net of Fig. 4. One remarks obviously that solving a reachability problem between markings $m_{0}=\left\{p_{1}, p_{2}, p_{3}, m_{1}, m_{2}, m_{3}\right\}$ and $m_{f}=\left\{p_{4}, p_{5}, p_{6}, m_{1}, m_{2}, m_{3}\right\}$ means exactly finding a schedule of the production presented in the table on the left side.

Production To Schedule:

job 1:

$$
\left(m_{1}, 2\right)\left(m_{2}, 3\right)\left(m_{3}, 4\right)
$$

job 2:

$$
\left(m_{2}, 3\right)\left(m_{3}, 2\right)\left(m_{1}, 3\right)\left(m_{3}, 3\right)
$$

job 3:

$$
\left(m_{1}, 2\right)\left(m_{3}, 4\right)\left(m_{1}, 2\right)
$$

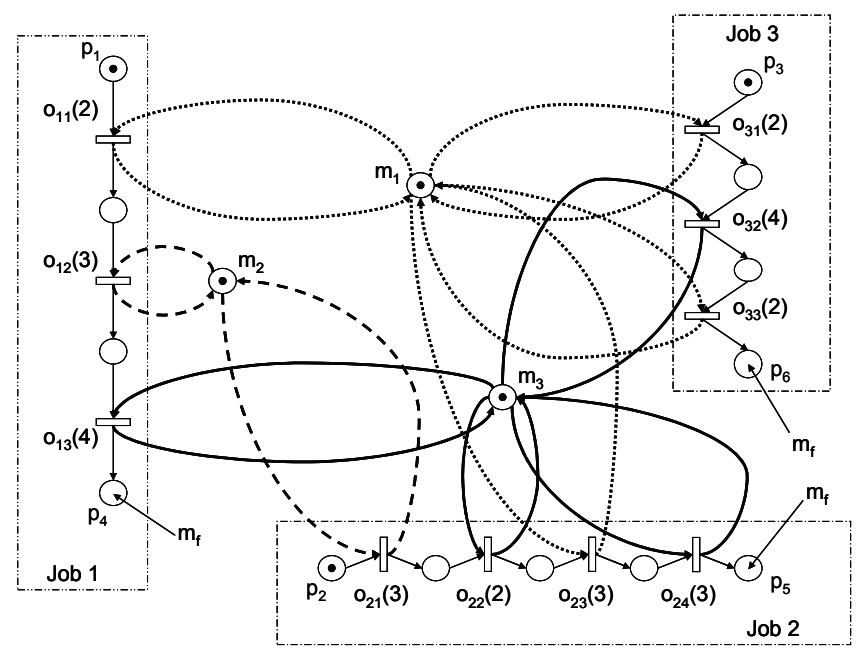

Fig. 4. TPN modelling a Production To Schedule 
Several approaches have been proposed to solve the Timed Petri net reachability problem, either by restricting their study to a subclass of TPN, like Timed Event Graphs (where a place has exactly one input and one output transition), either by using dedicated heuristics. A complete bibliography can be found in (Richard, 2000).

Since the fire of a Timed transition can occur as soon as it is fireable and as late as one wants, there may exist, from a given state, an infinite number of reachable markings (depending on the time), and no reachability graph can be built. A first approach needs to consider Timed $\mathrm{PN}$ as a subclass of Time PN, in order to use the state enumeration methods (state class graphs) proposed by (Berthomieu and Diaz, 1991). On the other hand, when dealing with early semantics (a transition is fired as soon as it is fireable), it is possible to proceed to an enumerative and structural analysis (David and Alla, 1992).

The early semantics has been extensively studied for the special class of Timed Event Graphs, using $(\max ,+)$ algebra (Bacceli et al., 1992). Since their structure does not handle conflicts, it is possible to obtain linear equations corresponding to the complete behaviour of the net.

In the following, we will show that our incremental approach can lead to mathematical programming models in the most general case.

\section{Incremental approaches}

As said before, the state equation (3) does not bring enough information to solve the reachability problem in all cases. This comes from the fact that it does not take into account the fireability conditions (1) of the individual transitions fired in the sequence $\sigma$. Incremental approaches improve this formulation by considering a given number of step firings corresponding to parallel and reentrant transitions. In this section, we discuss the interest of using steps and a fixed depth formulation.

\subsection{Step based reachability formulation}

Definition 14 (Step). Let $R$ be a Petri net. A step (Janicky and Koutny, 1991) is a multiset over the set of transitions $\mathrm{T}$. We denote by $\mathrm{T}^{*}$ the set of steps built over $\mathrm{T}$.

Informally, a step is a set that can contain several copies of the same element, e.g. $\left\{t_{1}, t_{1}, t_{2}\right\}$, which we would note hereafter simply $2 \cdot t_{1}+t_{2}$. We associate a step $\varphi=\sum_{j=1}^{N} \alpha_{j} \cdot t_{j}$ and its Parikh vector $\vec{\varphi}$ in the classical way, as a linear combination with non negative integer coefficients $\alpha_{j}$ of the Parikh vectors of each transition, i.e. $\vec{\varphi}=\sum_{j=1}^{N} \alpha_{j} \cdot \overrightarrow{e_{t_{j}}}$. A step is said empty, when $\varphi=\varnothing$, i.e. when $\forall j \in \square 1, N \square, \alpha_{j}=0$.

Note that a step can contain the same transition more than once, corresponding to transition reentrance. Thus, when working with Timed Petri nets, steps would only mean that several different transitions are considered to be fired at the same time.

For a step to be fireable, its preceding marking must contain enough tokens so that each transition of the step may consume its own tokens, as described in the following definition. 
Definition 15 (Step Firings). Let $R$ be a Petri net, $m$ be a marking and $\varphi$ be a step. The step $\varphi$ is fireable from $m$ iff:

$$
\vec{m} \geq C^{-} \cdot \vec{\varphi}
$$

If this condition is satisfied, the new marking $m^{\prime}$ reached from $m$ by the firing of $\varphi$ is defined as:

$$
\overrightarrow{m^{\prime}}=\vec{m}+C \cdot \vec{\varphi}
$$

Hereafter, we will use the notations already used previously: $m[\varphi\rangle, m_{0}[\varphi\rangle m_{1}$, $m_{0}\left[\varphi_{1} \varphi_{2} \ldots \varphi_{k}\right\rangle$ and $m_{0}\left[\varphi_{1} \varphi_{2} \ldots \varphi_{k}\right\rangle m_{k}$ to denote that a step or a step sequence is fireable, and the marking obtained in each case. The number of steps of a step sequence $\Phi=\varphi_{1} \varphi_{2} \ldots \varphi_{K}$ is denoted by $|\Phi|=K$.

The definition of step firings corresponds naturally to the firing of the underlying transitions. We will show that its use can lead to a formulation that is still equivalent to the initial PN behavior, but that can be more conveniently used in a mathematical programming framework. The following proposition explains the relation between step and transition firings with respect to reachability issues.

Proposition 2 (Step Reachability Equivalence). Let $\left(R, m_{0}\right)$ be a Petri net and $m_{f} a$ marking.

$m_{f}$ is reachable from $m_{0} \Leftrightarrow$

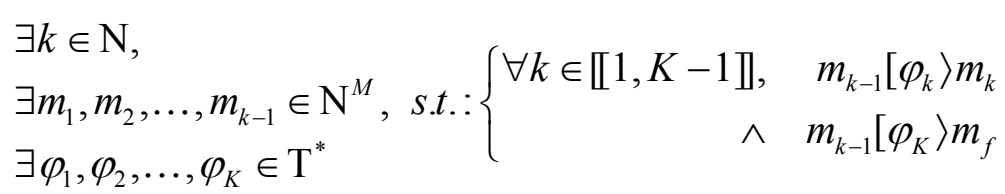

Proof. The proof of this proposition is not difficult but quite lengthly and hence is not given in this chapter. It can be found in a technical report available at url: http://www.eclille.fr/tomnab/asr07/. W

One must remark that the proof of the proposition 2 shows how it is possible to construct a firing sequence leading to $m_{f}$ from a corresponding step sequence. Thus, to compute a firing sequence leading to a target marking, it will be sufficient to compute a step sequence leading to the same marking.

The main interest of our formulation is to capture the parallelism caused by the interleaving of actions, which is precisely one of the main advantages of using a Petri net as a model of a system. This issue has already been followed by (Vernadat et al., 1996), from whom we borrow the illustrative example of Fig. 5: "When two ( $n$ in the general case) components offer independent actions in parallel, the interleaving semantics expresses this behaviour by a diamond (an hypercube in the general case) within which each path lead to the same final state. Since all paths 
converge to the same state, the key idea is to develop only one particular path among the set of possible equivalent ones".

Example 3 (Vernadat's steps). As shown in Fig. 5, there exists several ways to handle 3 independent transitions from the point of view of the reachability graph. One can consider them one by one, which leads to handle 8 states and 12 firings. If we just refer to their corresponding Mazurkiewicz's trace (see (Vernadat et al., 1996) for details), we only have to handle 3 transition firings and 4 states. In the last case, one can capture the whole behavior in one unique firing that is called a step by Vernadat (with the meaning of "footstep").

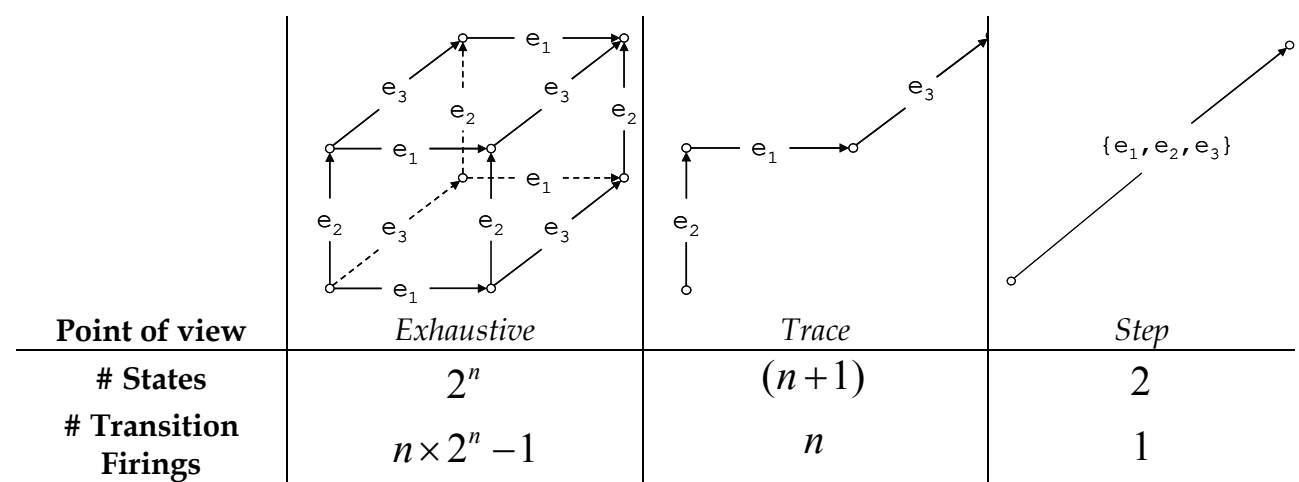

Fig. 5. Some ways to handle independent transitions, from Vernadat

The characterization given in proposition 2 can be used to build a mathematical programming model based on steps which can be used to solve reachability-based PN analysis problems: one has just to express the right side of equation (10) using the linear equations (8) and (9) over integer variables. Such a model will be presented in section 4 .

The advantage of using steps is that they allow to reduce the number of firings in our model - and then the number of variables - while keeping an equivalence with the initial properties. Thus it is not a modification of the semantics of PNs, but only a way to capture the independence of transitions. Of course, this reduction does not systematically holds, since it is easy to construct a Petri net where only one transition can be fired at a time. Thus, in the worst of cases, the step firings formulation may not bring any improvement as far as the number of firings used are concerned. However, this is a quite uncommon situation since it means that the Petri net does not show any parallelism.

\subsection{Incremental search}

We have seen the interest of using steps to formulate the reachability problem in PNs as a search for instanciations of integer variables constrained by a system of linear equations. This formulation allows us to use the paradigm of mathematical programming to solve the reachability problem. However, the initial definition of the reachability problem is not well adapted to the kind of formulation we propose to use, since definition 5 does not make any assumption concerning the number of steps needed to solve the reachability problem. In this paragraph, we define two sub-problems associated with the original reachability problem introduced before, which can be conveniently solved using the characterization of proposition 2 in a mathematical programming framework. 
Definition 16 (Fixed Depth Reachability Problem). Let $\left(R, m_{0}\right)$ be a Petri net, $k \in \mathrm{N}$ and $m_{f}$ a marking.

$\mathrm{P}_{1}(k) \quad$ Find a step sequence allowing to reach the marking $m_{f}$ from the marking
$m_{0}$ in at most $k$ steps.

Definition 17 (Shortest Length Reachability Problem). Let $\left(R, m_{0}\right)$ be a Petri net, and $m_{f}$ a marking reachable from $m_{0}$.

$$
\begin{aligned}
& \mathrm{P}_{2} \quad \text { Find the minimal length, denoted by } K_{\min } \text {, of a sequence of steps allowing to } \\
& \text { reach the marking } m_{f} \text { from the marking } m_{0} .
\end{aligned}
$$

Of course, each of these sub-problems is directly linked to the initial one defined before, and each allows to solve a different kind of PN reachability analysis. For instance, the first formulation $\mathrm{P}_{1}(k)$ is highly useful for model-checking since it can serve to define an exhaustive search of the reachability graph. On the other hand, the second formulation $\mathrm{P}_{2}$ is well designed to deal with performance analysis since it returns a firing sequence that maximizes the parallelism of the system. It can also give an helpful bound for the definition of additional heuristics. Finally, since it is clear that the complexity of the problem grows (w.r.t. number of variables and constraints) as the length $k$ of the sequence of steps used increases, it seems also quite reasonable to search for the smallest value of the parameter $k$ from which a solution exists.

The fixed depth reachability problem $\mathrm{P}_{1}(k)$ has already been studied by (Benasser, 2000) using the logical abstraction technique. His approach is based on the same notion of steps, but it uses constraint programming techniques. His algorithm iterates the number of steps used, adding one new step at each iteration, in order to test all the lengths of sequences of steps lower than $k$. Benasser proved that his algorithm is correct since the sequences found are effectively sequences of steps which produce the desired final marking. It is also complete since it can enumerate all the solutions of length smaller than a given integer $k$. In each iteration, the algorithm uses a mechanism of linear constraints solving. It has been implemented using the constraint logic programming software Prolog IV. The interest of using a constraint logic programming framework is that its resolution mechanism is incremental (Jaffar et al., 1992). Indeed, it is not necessary to redefine in each iteration the constraints incorporated into the previous stage. The constraints are added in the constraints solver so that it can reuse the results of the previous constraints propagation. The search for the concrete results is made at the end by an enumeration of all the possible integer solutions, which corresponds exactly to the sub-problem formulation $\mathrm{P}_{1}(k)$.

In section 4, we will adapt Benasser's algorithm to our own mathematical programming framework. To achieve the same kind of results, we will prove the correctness and completeness of our mathematical programming formulation with respect to $\mathrm{P}_{1}(k)$. These results will allow us to use integer linear programming techniques to find every solution of 
$\mathrm{P}_{1}(k)$. Some objective functions would also be defined to guide the search directly to an optimal solution in some way. Since $\mathrm{P}_{2}$ can be easily expressed by iterating $\mathrm{P}_{1}(k)$ instances for growing values of the parameter $k$, it will also be solved using the same technique.

Here, Operational Research techniques replace Artificial Intelligence ones, but the algorithm structure is the same. All techniques based on incremental approaches may share the same search algorithms. The most basic algorithm consists in searching in an incremental way amongst sequences the length of which are increased one by one.

\subsubsection{Naive algorithm}

This algorithm is fed with a bound $K_{\max }$ on what we call the "search depth" in order to prevent an infinite loop. Once chosen this value, the procedure generates iteratively a sequence of mathematical models of increasing size, and search for solutions in the corresponding search spaces using mathematical programming techniques. If there is no solution in less than $k_{\max }$ steps, the algorithm stops. It is described in Fig. 6.

1: $\quad k \leftarrow 0$

2: DO

3: $\quad k \leftarrow k+1$

4: $\quad$ Generate $\mathrm{MP}(k)$, a mathematical programming model for the problem $\mathrm{P}_{1}(k)$ (which corresponds to characterization of proposition 2 with $k$ steps ).

5: $\quad$ Solve the model $\mathrm{MP}(k)$ using branch \& bound techniques (e.g. Cplex solver). Let $\vec{X}_{i \llbracket 1, k \rrbracket}$ be an optimal solution of $\operatorname{MP}(k)$ if it exists.

6: $\quad$ IF $(\operatorname{MP}(k)$ has a solution $)$, RETURN $\vec{X}_{i \llbracket 1, k \rrbracket}$

7: $\quad$ WHILE $(\mathrm{MP}(k)$ is infeasible $)$ AND $\left(k \leq K_{\max }\right)$

Fig. 6. Naive Search Algorithm

During the formulation of the mathematical programming model at step 4 , one should take care of the domain of variables representing the steps. Indeed: the definitions of step and step firings do not forbid empty steps leaving the markings unchanged. By considering empty steps valid in our formulations, we get the following result.

Proposition 3 (Satisfaction Monotony) Let $k \in \mathrm{N}$. If the problem $\mathrm{P}_{1}(k)$ is feasible, then for any integer $k^{\prime} \geq k$, the problem $\mathrm{P}_{1}\left(k^{\prime}\right)$ is also feasible.

Proof. It is easy to construct a feasible solution for $\mathrm{P}_{1}\left(k^{\prime}\right)$ from a feasible solution of $\mathrm{P}_{1}(k)$ for $k^{\prime} \geq k$ by adding empty steps. W

Note the same result would be true when dealing with the family of mathematical programming models $\operatorname{MP}(k)$ : if there exists $k \in \mathrm{N}$ such that $\operatorname{MP}(k)$ admits a solution, any model $\mathrm{MP}\left(k^{\prime}\right)$ with $k^{\prime} \geq k$ would be feasible too. This property motivates the jump search techniques proposed in the next paragraph. 


\subsubsection{Jump search}

From proposition 3 and the definition of parameter $K_{\min }$, we get:

$$
\left\{\begin{array}{lc}
\forall k<K_{\text {min }} & \mathrm{P}_{1}(k) \text { is infeasible } \\
\forall k \geq K_{\text {min }} & \mathrm{P}_{1}(k) \text { is feasible }
\end{array}\right.
$$

This property can help us to define new iterative techniques, since - for example - it shows that it is not necessary to solve all the problems $\mathrm{P}_{1}(k)$ for $k \leq K_{\min }$, like in the naïve search described before.

Of course, as said before, we must keep using an incremental procedure in order to avoid the use of large models if they are not needed. We propose finally some techniques based on jumps over the values of the search depth. These techniques allow to decrease the number of iterations needed, thus improving the search efficiency. Several jump strategies are possible. We describe briefly some elementary ones.

- Forward jump search The first family continously increases the value of the search depth. We can distinguish two main politics, depending on how the amplitude of jumps is defined.

- $\quad$ Fixed amplitude Its value must be chosen in order to obtain a high exploration speed while minimizing the possible redundant steps. This type of strategy allows to estimate the profit precisely.

- $\quad$ Dynamic amplitude This second strategy uses variable amplitudes. Increasing amplitudes should be used for small values of $k$, and decreasing ones when the exploration becomes more difficult. This kind of behavior is less easily quantifiable.

These politics both can lead to overtake $K_{\text {min }}$ when a solution is found. In this case, it is not anymore possible to answer precisely the problem $\mathrm{P}_{2}$, since we do not get the exact value of $K_{\min }$. To compensate this lack of information, one can use a dichotomic search.

- Dichotomic search This kind of procedure needs to know a maximal bound for $k$. Its value is given by a previous successful execution of the forward jump search.

The main interest of jump search is that it allows to win in efficiency. Since we do not know the number of steps needed to find a solution if it exists, the use of such a technique allows us, when it is possible, not to have to develop the entire set of formulations of length lower than $K_{\min }$. Numerical experiments show that even if the size of models is increasing, the corresponding practical complexity does not always follows the same evolution.

Finally, it must be said that the procedure described in Fig. 6 is only a semi-complete one. Indeed, in the context of unbounded PNs, the value of $k_{\max }$ is set arbitrarily, as we do not know any information on the number of steps needed to find a possible solution. Thus, if no solution is obtained before the value of $k$ has been reached, one cannot conclude on the reachability property. 
To the contrary, when dealing with bounded PNs, it is possible to set $K_{\max }$ to the value of the sequential depth of the net, a parameter we have defined in (Bourdeaud'huy et al., 2004a) and which guarantee the complete exploration of the reachability graph. Using this parameter as search depth, it is always possible to conclude when the algorithm stops.

\subsection{Adaptation to timed Petri nets}

We have seen in the previous section the awaited benefits from using an incremental approach made of step firings. Before introducing the mathematical models in section 4, we propose to adapt the step based formulation to Timed Petri nets.

We start by adapting the previous formalism to Timed Petri nets. The key idea is again to consider the evolution of a Timed Petri net "step by step".

Definition 18 (Timed step). Let $(R, d)$ be a Timed Petri net. A timed step is a pair $\psi=(\varphi, v)$ such that:

- $\varphi=\sum_{j \in \llbracket 1, n \rrbracket} \alpha_{j} \cdot t_{j}$ is a step $\in \mathrm{T}^{*}$ for the Place/Transition Petri net $R$, such that $\forall j \in \square 1, N \square, \alpha_{j} \in\{0,1\}$;

- $v$ is a date $\in \mathrm{N}$.

The set of all timed steps of a Timed Petri Net is denoted by $\mathrm{T}_{\mathrm{TPN}}^{*}$.

Definition 19 (Timed steps Firings). Let $(R, d)$ be a Timed Petri net. Let $e=\left(\overrightarrow{E_{m}}, \overrightarrow{E_{r}}\right)$ be a state given at date $v$. Let $v^{\prime} \geq v$ and $\Delta_{v}=v^{\prime}-v \in \mathrm{N}$. The timed step $\psi=\left(\varphi, v^{\prime}\right)$ is fireable from e iff:

$$
\begin{gathered}
\forall t \in \varphi,\left.\overrightarrow{E_{r}}\right|_{t} \leq \Delta_{v} \\
C^{-} \cdot \vec{\varphi} \leq \overrightarrow{E_{m}}+C^{+} \cdot \sum_{\substack{t \in \mathrm{T}, 0<\left.\overrightarrow{E_{r}}\right|_{t} \leq \Delta_{v}}} \overrightarrow{e_{t}}
\end{gathered}
$$

If this condition is satisfied, the new state $e^{\prime}=\left(\overrightarrow{E_{m}^{\prime}}, \overrightarrow{E_{r}^{\prime}}\right)$ reached at date $v^{\prime}$ from $e$ by the firing of $\psi=\left(\varphi, v^{\prime}\right)$ is defined as:

$$
\begin{aligned}
& \overrightarrow{E_{m}^{\prime}}=\overrightarrow{E_{m}}-C^{-} \cdot \vec{\varphi}+C^{+} \cdot \sum_{t \in \mathrm{T},} \overrightarrow{e_{t}} \\
& 0<\left.\overrightarrow{E_{r}}\right|_{t} \leq \Delta_{v} \\
& \forall t \in \mathrm{T},\left.\overrightarrow{E_{r}^{\prime}}\right|_{t}=\left\{\begin{array}{c}
d(t) \quad \text { if } t \in \varphi \\
\left.\overrightarrow{E_{r}}\right|_{t}-\Delta_{v} \quad \text { if }\left.\overrightarrow{E_{r}}\right|_{t}-\Delta_{v}>0 \\
0 \quad \text { otherwise }
\end{array}\right.
\end{aligned}
$$


The above definition follows the firing semantics of Timed Petri nets described above, from the point of view of a punctual firing between two markings. Informally, equation (12) means that a transition fired within a step must not be active at the time of the firing, in order to comply with the non-reentrance hypothesis. Equation (13) verifies that there is enough tokens at date $v^{\prime}$ to fire the step $\varphi$. The set $\mathrm{A}=\left\{t \in \mathrm{T}, 0<\left.\overrightarrow{E_{r}}\right|_{t} \leq \Delta_{v}\right\}$ used in equations (13) and (14) denotes the transitions that were active at date $v$ and are no longer alike at date $v^{\prime}$. Thus, the quantity $\sum_{\mathrm{A}} C^{+} \cdot \overrightarrow{e_{t}}$ corresponds to the tokens that would appear between dates $v$ and $v^{\prime}$. At last, the update of the residual durations vector at equation (15) is made as follows:

- If the transition $t$ is fired in the step $\varphi$, its duration is used to initialize the corresponding component of the residual vector;

- If a transition that was previously active is still active at date $v^{\prime}$, its residual duration is updated by taking into account the time elapsed from the previous date;

- Otherwise, if a transition was active and has finished, or if a transition was not active and is not fired in the step $\varphi$, its residual duration is null.

As above, we will use the notations $e[\psi\rangle, e_{0}[\psi\rangle e_{1}, e_{0}\left[\psi_{1} \psi_{2} \ldots \psi_{k}\right\rangle$ and $e_{0}\left[\psi_{1} \psi_{2} \ldots \psi_{k}\right\rangle e_{k}$ to indicate that a timed step or a timed step sequence is fireable, and the state obtained in each case. We give finally below the main proposition concerning the use of timed steps in the context of Timed Petri nets.

Proposition 4 (Equivalence between Controlled Executions and timed step firings). Let $(R, d)$ be a TPN with its initial state $e_{0}=\left(\overrightarrow{E_{m_{0}}}, \overrightarrow{0_{N}}\right)$ given at date $v_{0}=0$. Let $e_{f}=\left(\overrightarrow{E_{m_{f}}}, \overrightarrow{0_{N}}\right)$ be a state.

There exists a feasible controlled execution allowing to reach $e_{f}$ at date $v_{\max }$ from $e_{0} \Leftrightarrow$

$$
\begin{aligned}
& \exists k \in \mathrm{N}, \\
& \exists v_{1}, v_{2}, \ldots, v_{K} \in \mathrm{N} \\
& \exists e_{1}, e_{2}, \ldots, e_{K} \in \mathrm{S}(R, d) . \\
& \exists \psi_{1}=\left(\varphi_{1}, v_{1}\right), \psi_{2}, \ldots, \psi_{K}=\left(\varphi_{K}, v_{K}\right),
\end{aligned} \quad \text { s.t.: }\left\{\begin{array}{r}
\forall k \in \square 1, K \square, e_{k-1}\left[\psi_{k}\right\rangle e_{k} \\
e_{K}\left[\psi_{\text {max }}\right\rangle e_{f}
\end{array}\right.
$$

Proof. Here again, the reader is referred to the technical report available at http://www.eclille.fr/tomnab/asr07/ for the complete proof. W Following the previous proposition, it is sufficient to search for timed step sequences to solve the reachability problem in TPN. The advantage of such an approach is obvious: like for basic PN, it is well adapted to the definition of a mathematical programming model with a reduced number of variables and constraints, since it allows to consider explicitly parallel 
executions. Moreover, it is incremental since one can progressively increase the number of steps used in the formulation without redefining the whole set of constraints.

Since reachability by controlled execution or timed step sequence is equivalent, we define as above a sub-problem of the TPN reachability problem, which can be conveniently solved using the characterization of proposition 4 in a mathematical programming framework.

Definition 20 (Fixed Depth Timed PN Reachability Problem). Let $(R, d)$ be a TPN with its initial state $e_{0}=\left(\overrightarrow{E_{m_{0}}}, \overrightarrow{0_{N}}\right)$ given at date 0 . Let $e_{f}=\left(\overrightarrow{E_{m_{f}}}, \overrightarrow{0_{N}}\right)$ be a target state.

$$
\begin{aligned}
& \mathrm{TP}_{1}(v) \quad \text { Find a timed step sequence allowing to reach the state } e_{f} \text { from the state } e_{0} \\
& \text { in at most } v \text { timed steps. }
\end{aligned}
$$

In the next section, we prove the correctness and completeness of our mathematical programming model with respect to this formulation.

\section{Integer linear programming models}

In this section, we give integer linear programming models corresponding to the characterizations introduced in propositions 2 and 4.

\subsection{Place/transition Petri nets}

\subsubsection{Integer linear programming model}

In the previous section, we have shown that step sequences are sufficient to prove that a marking is reachable and to find the firing sequence leading to it. In this section, we show how the search for a step sequence can be expressed as a mathematical programming problem. We prove also that this model is correct and complete with respect to the fixed depth reachability problem $\mathrm{P}_{1}(k)$.

Our integer programming model is directly built from equations and inequalities (8), (9) and (10). Thus we get:

Model 1 (Integer Programming Model). Let $\left(R, m_{0}\right)$ be a Petri net with $\mathrm{P}=\left\{p_{1}, \ldots, p_{M}\right\}, \mathrm{T}=\left\{t_{1}, \ldots, t_{N}\right\}, m_{f}$ a marking and $K \in \mathrm{N}$. The integer linear program $\operatorname{IP}(K)$ is defined by:

$$
\begin{gathered}
\text { Minimize } \omega(\vec{X}) \\
\text { subject to: } \\
\operatorname{IP}(K) \quad \forall i \in \llbracket 1, K \rrbracket, \sum_{j=1}^{i-1} C \cdot \overrightarrow{X_{j}}-C^{-} \cdot \overrightarrow{X_{i}} \geq-\overrightarrow{M_{0}} \\
\sum_{i=1}^{K} C \cdot \overrightarrow{X_{i}}=\overrightarrow{M_{f}}-\overrightarrow{M_{0}} \\
\forall i \in \llbracket 1, K \rrbracket, \quad \forall j \in \llbracket 1, N \rrbracket, \quad X_{i j} \in \mathrm{N}
\end{gathered}
$$

In the model $\operatorname{IP}(K)$, variables $X_{i j}$ represent the components of $K$ steps in the sense of definition 14. Inequalities $(17 b)$ correspond to the combination of fireability (8) and 
reachability (9) conditions presented in definition 15 . To reduce the size of the problem, the variables $\left(m_{i}\right)_{\llbracket 1, K \square}$ of equation (10) have been dropped by using the substitution of equation (9). Equation (17c) corresponds to the target marking to reach like in (10). The integrality constraints of variables $X_{i j}$ are expressed by (17d). The expression of objective function $\omega(\vec{X})$ will be described later.

The following proposition is central in our construction: it shows that our model $\operatorname{IP}(k)$ characterizes all the solutions of the problem $\mathrm{P}_{1}(k)$.

Proposition 5 (Correctness and completeness of $I P(k)$ w.r.t. $\left.P_{1}(k)\right)$. Let $\left(R, m_{0}\right)$ be a PN and $k \in \mathrm{N}$. Then we have:

- Any solution of $\operatorname{IP}(k)$ is also a solution of $\mathrm{P}_{1}(k)$ (Correctness)

- Any solution of $\mathrm{P}_{1}(k)$ can be expressed as a solution of $\operatorname{IP}(k)$ (Completeness)

Proof. Those results come directly from the proof of proposition 2.

According to proposition 5, to solve the fixed-depth PN reachability problem is equivalent to search for the solutions of an integer linear programming problem. In this way, the exploration of the reachability graph and the resolution of the corresponding reachability problems are reduced to the resolution of a system of equations. The "combinatorially explosive" reachability graph is reduced to a sequence of "abstract" steps. Since $\operatorname{IP}(k)$ needs $k . n$ variables and $(k+1) \cdot M$ constraints, the size of the $\operatorname{IP}(k)$ model grows linearly with the value of the parameter $k$. This having been said, two remarks must be done.

- Of course, we do not pretend that the combinatorial explosion problem has been wiped out. It is only postponed to the mathematical programming solution phase. Meanwhile, our formulation allows to benefit automatically from today's best solvers.

- On the other hand, compared to many other exploration techniques, one of the interests of our formulation is to avoid the "exploration" of the branches of the graph which do not lead to the desired final marking. Indeed, these cases are "automatically cut off" by the equation (17c).

From these two statements, we have good reasons to think that our method will bring some improvements on the research topic considered. We have developed several additional mechanisms to validate our approach and to improve the performance of the solution. All these improvements have been formally defined in (Bourdeaud'huy et al., 2004a,b,c, 2007; Bourdeaud'huy, 2004). We describe them below and refer the reader to these papers for more information.

\subsubsection{Practical improvements}

Objective Functions: It is obvious that $\mathrm{P}_{1}(k)$ has a solution if and only if the feasible set of $\mathrm{IP}(k)$ problem is non empty. This property stays true for any objective function. 
Nevertheless, the efficiency of solving $\operatorname{IP}(k)$ may depend on the objective function chosen. Let's remark that some objective functions are more usefull in practice than others.

For example, if there is no difference between solutions, a constant function is valid, which leads to the selection of the first feasible solution found. We define thus the function vanishing identically:

$$
\forall \vec{X}, o b j_{1}(\vec{X})=0
$$

This objective function leads to the selection of the first feasible solution found, thus to the best practical performances. We could also use physical sense objective functions according to the particular context of the studied problem. For example, we could differentiate the solutions by their norm, considering thus the $L_{1}$ norm of steps:

$$
\forall \vec{X}, o b j_{2}(\vec{X})=\sum_{i=1}^{K} \mathrm{P} \overrightarrow{X_{i}} \mathrm{P}_{1}=\sum_{i=1}^{K} \sum_{j=1}^{N} X_{i j}
$$

The function $\mathrm{obj}_{2}$ allows to search for the "fastest" sequence, in terms of number of firings.

Relaxations: In order to decrease the time needed to conclude on the infeasibility of the $\mathrm{IP}(k)$ problem, we propose to use relaxation techniques. They are useful in the field of combinatorial optimization. The principle of these techniques is to replace the complex original problem by one or several simpler ones.

Definition 21 (Relaxation). A relaxation of an optimization problem $P$ of type maximisation is an optimization problem $R$ such that:

- Each feasible solution for $P$ is also a feasible one for $R$;

- The value of the objective function of any solution of $R$ is greater than or equal to the value of the objective function of the same solution for $P$.

Among useful properties of the relaxation and duality techniques in solving an optimization problem $P$ is that the optimal value of the relaxation problem provides an upper bound on the optimal value of the corresponding $P$. Moreover, if the relaxed problem is infeasible then the problem $P$ is also infeasible. In our context, this second property is used to conclude that the $\operatorname{IP}(k)$ problem is infeasible by solving a relaxed problem before reaching $K_{\min }$.

- LP-relaxation consists in relaxing integrality constraints. Bounds derived from other relaxations can be stronger than those obtained from $L P$. In the literature (Parker and Rardin, 1988), Lagrangean relaxation, surrogate relaxation and composite relaxation are usually used to obtain such upper bounds.

- Lagrangean relaxation consists to relax complicating contraints and incorporating them into the objective function with a so-called Lagrangean multiplier (Geoffrion, 1974). However, note that relaxing fireability $(17 \mathrm{~b})$ or reachability $(17 \mathrm{c})$ constraints is "too strong" from the physical point of view. Indeed: without fireability conditions, the modified model represents only the state equation, which is already supposed to have 
solutions. In the other hand, dealing with a model without the reachability constraint correspond to study the whole behaviour of the net. Any sequence of fireable transitions of the correct length is solution to the relaxed problem and do not bring information.

- Surrogate relaxation replaces the original constraints by a single new one, called a surrogate constraint (Glover, 1977).

Binary Programming Model: We propose also a binary programming model denoted by $\mathrm{BIP}(K)$. In this model, equation $(17 \mathrm{~d})$ is replaced by:

$$
\forall i \in \square 1, K \square, \forall j \in \square 1, N \square, X_{i j} \in\{0,1\}
$$

This formulation is still correct since it corresponds to a restriction of the initial model $\mathrm{IP}(K)$ : parallel behaviours are still allowed, but reentrance is forbidden. This formulation may be more efficient since the domain of variables is reduced. However, it may be necessary to fire more steps to reach some markings than using the $\operatorname{IP}(K)$ model. Nevertheless, it is quite simple to show that this last model preserves an equivalence with the $\operatorname{IP}(K)$ one. Indeed, any solution of $\mathrm{BIP}(K)$ is also obviously a solution of $\operatorname{IP}(K)$. Inversely, any solution of $\operatorname{IP}(K)$ could be obtained by an aggregation of a solution of $\operatorname{BIP}\left(K^{\prime}\right)$, with $K^{\prime} \geq K$ (some steps in a solution of $\operatorname{IP}(K)$ may have to be splitted into several firings of what could be called "binary steps" of the solution from $\left.\operatorname{BIP}\left(K^{\prime}\right)\right)$. Thus we are able to use the model $\operatorname{BIP}\left(K^{\prime}\right)$ in the same way as $\operatorname{IP}(K)$. The only difference is the value of $K_{\text {min }}^{\prime}$ associated to $\operatorname{BIP}\left(K^{\prime}\right)$ which can be greater than $K_{\text {min }}$.

Empty Steps Management: As said before, to consider empty steps valid in our formulations bring interesting theoretical results. However, practically speaking, empty steps do not bring useful information considering the resolution of the reachability problem, since they do not change the markings. We propose thus several additional constraints dedicated to the management of empty steps .

- In the binary model, one can add an extra linear constraint in order to express a notion of partial order in the steps:

$$
\forall i \in \square 1, K-1 \square, N \cdot \sum_{j=1}^{N} X_{i j} \geq \sum_{j=1}^{N} X_{(i+1) j}
$$

These constraints mean that empty steps have to appear at the end of the constrained sequence. Indeed: if a step $X_{i}$ is empty (i.e. $\forall j \in \square 1, N \square, X_{i j}=0$ ), all its successors $\left(X_{k}\right.$ s.t. $\left.k \in \square i+1, K \square\right)$ in the step sequence have to be empty in a "chain reaction". Inversely, since we consider binary steps, equation (19) is obviously true when $\exists j \in \square 1, N \square$ s.t. $X_{i j}=1$, because then $N \cdot \sum_{j=1}^{N} X_{i j} \geq N$. 
- We propose also a new objective function, which corresponds to maximize the number of empty steps in the feasible sequence. This is equivalent to minimize the number of nonempty steps $\mathrm{Obj}_{3}$ defined below:

$$
\forall \vec{X}, \operatorname{obj}_{3}(\vec{X})=\operatorname{card}\left\{i \in \llbracket 1, K \rrbracket \text { s.t. } \sum_{j=1}^{N} X_{i j} \neq 0\right\}
$$

The function $\mathrm{Obj}_{3}$ allows us to look for the shortest solutions in term of "equivalent length" when the empty steps have been removed. To model $o b j_{3}$, we introduce new intermediate binary variables $\alpha_{1}, \alpha_{2}, \ldots, \alpha_{k}$ and we incorporate in the linear programming models the following additional constraints:

$$
\begin{array}{cc}
\forall i \in \llbracket 1, K \rrbracket, & 1+\left(\alpha_{i}-1\right) \cdot B \leq \sum_{j=1}^{N} X_{i j} \leq \alpha_{i} \cdot B \\
\forall i \in \llbracket 1, K \rrbracket, & \alpha_{i} \in\{0,1\}
\end{array}
$$

where $B$ is a sufficiently big number, chosen much bigger than the number of transitions and tokens in the net, e.g. $B \geq M \cdot N \cdot K$. Since the variables $\left(\alpha_{i}\right)_{\square 1, K \square}$ are binary, it is easy to check that $\alpha_{i}=0$ iff the step $X_{i}$ is empty (a complete proof is given below in proposition 6). Thus the objective function $\mathrm{obj}_{3}$ can be expressed in the following way:

$$
\forall X, \operatorname{obj}_{3}(X)=\sum_{i=1}^{K} \alpha_{i}
$$

- Finally, one could simply forbid empty steps by adding to the models the following constraint:

$$
\forall i \in \llbracket 1, K \rrbracket, \mathrm{P} \overrightarrow{X_{i}} \mathrm{P}_{1} \geq 1
$$

Decomposition Technique: To improve the performance of the resolution, we have proposed constraints corresponding to a decomposition technique based on a partition of the state space according to the solutions of the PN state equation. For each solution $\vec{\sigma}$ of the underlying state equation - defined between the same initial and final markings -, we add the constraint:

$$
\forall j \in \llbracket 1, N \rrbracket, \sum_{i=1}^{K} X_{i j}=\left.\vec{\sigma}\right|_{t_{j}}
$$


Such a decomposition technique allows to adress the complexity of the problem in two steps:

- The first step uses well known T-invariants computation techniques (see for example (Colom and Silva, 1989a)) which are independent from the initial marking, and thus allows to reuse the same information for many different initial and final markings.

- Given the Parikh vector of the whole firing sequence to discover, the resolution of the reachability problem should be slighty simple. However, one should note that this second step remains difficult: it is not sufficient to distribute the firings over the steps since each step must be fireable. Moreover, developping heuristics methods is challenging since deadlock situations can occur late after a bad choice has been made. Finally, the mathematical programming approaches proposed here are well designed to handle the second step of the search.

\subsubsection{Numerical experiments}

Numerous practical experiments were led in (Bourdeaud'huy et al., 2004a,b,c, 2007; Bourdeaud'huy, 2004) in order to assess the efficiency of our mathematical programming models. There is no space left to copy them all here, but several results must be pointed out.

- We have compared the influence of the objective functions $o b j_{1}, o b j_{2}$ and $o b j_{3}$. The corresponding results were quite foreseeable: $o b j_{1}$ led to the best results, followed by $o b j_{2}$ and finally $o b j_{3}$. However, it must be said that the performance of resolution using $\mathrm{Obj}_{3}$ was very weak, even for the smallest instances of our families, since the size of the corresponding models is large. To the contrary, the performance of models using $o b j_{2}$ were quite close to the basic performance given by $o b j_{1}$, about 3 times worse in a pinch.

- Our experiments to validate the pertinence of the LP-relaxation shown that for the whole set of PN studied, the gap between $K_{\text {min }}$ for the LP-relaxation and the integer model is small. Such statement suggests a property of integrality of the kind of problems considered - i.e. continuous solutions are somehow integer -, which may be interesting to study.

- Compared one with another, model BIP behaves better than IP. Of course, one could build an example for which the contrary would holds. Nevertheless, this observation were made over the whole set of our experiments.

- Dealing with decomposition technique, preliminary experiments have shown that a search inside a given equivalence class is $20 \%$ faster than for the whole state space.

- We have compared our technique with other classical tools from the PN community: Ina (Roch and Starke, 2002) and Netched (Benasser, 2000).

- Ina means Integrated Net Analyzer. It is an analysis tool which allows the computation of firing sequences between markings thanks to the exploration of a covering graph. It implements some reduction techniques, e.g. persistent sets (Valmari, 1991) and symmetries (Schmidt, 1998).

- Netsched is the implementation of the logical abstraction technique developped by Benasser. It has been implemented using the constraint logic programming 
language Prolog IV.

Our approach has shown very good results and dominates the other tools on some instances. However, there exists some special instances - see for example (Bourdeaud'huy et al., 2004c) - for which our method is dominated by Netsched, particularly when the underlying reachability graph is sparse.

In the next section, we develop a similar mathematical programming approach for Timed Petri nets.

\subsection{Timed Petri nets}

We proceed as for Place/Transition PN, by adapating the characterization proposition 4 to build a mathematical programming model. For that, we need to linearize the equations defining timed step firings. We introduce in the next section two operators and the corresponding linearization variables and equations that we use to obtain the linear integer programming model.

\subsubsection{Discrimination operators}

We start by giving a useful proposition, which has been already used above dealing with the formulation of objective function $\mathrm{Obj}_{3}$.

Proposition 6 (Discrimination Variables). Let $X \in \mathrm{S} \subset \mathrm{Z}$ and $B \in \mathrm{N}^{*}$ be "sufficiently large". Let $\alpha \in\{0,1\}$ and $\beta \in \mathrm{N}$ such that:

$$
\begin{gathered}
1+(\alpha-1) \cdot B \leq X \leq \alpha \cdot B \\
X \leq \beta \\
\beta \leq \alpha \cdot B \\
\beta \leq B \cdot(1-\alpha)+X
\end{gathered}
$$

Then we have:

$$
\left\{\begin{array}{l}
X>0 \Rightarrow \alpha=1 \text { et } \beta=X \\
X \leq 0 \Rightarrow \alpha=0 \text { et } \beta=0
\end{array}\right.
$$

Proof. Let's assume that $X$ is strictly positive. The right side of inequation (20a) implies then $1 \leq X \leq \alpha \cdot B$, i.e. $\alpha \geq \frac{1}{B}>0$. Thus we have $\alpha=1$, and the left side of inequation (20a) is valid. The inequation (20d) implies then $\beta \leq X$, and inequation (20b) implies $\beta=X$. Inequation (20c) is valid only if $B$ is sufficiently large, namely: $B \geq \max _{X \in \mathrm{S}}(X)$. Conversely, if $X$ is negative or null, the left side of inequation (20a) implies $1+(\alpha-1) \cdot B \leq 0$, which implies $\alpha \leq 1-\frac{1}{B}<1$. We have then $\alpha=0$ and the right 
side of inequation (20a) is valid. The inequation (20c) implies then $\beta \leq 0$, i.e. $\beta=0$. Inequation (20b) is valid and inequation (20d) is valid if $B$ is sufficiently large, again if $B \geq \max _{X \in \mathrm{S}}(|X|)$.

W

Using 2 variables and 5 equations per unknown $X$, one can thus obtain linearly $X^{s}$ and $X^{+}$, corresponding to the "sign" of $X$ and its "positive component". We extend these operators " + " and " $s$ " to vector objects, by applying them uniformly on each component of the considered vector.

Definition 22 (Discrimination Operators). Let $k \in \mathrm{N}$ and $\vec{x} \in \mathrm{Z}^{k}$. We denote by:

- $\vec{x}^{+} \in \mathrm{N}^{k}$ the vector of its positive components, such that $\forall c \in \llbracket 1, k \rrbracket, \vec{x}(c)=\vec{x}(c)$ if $\vec{x}(c)>0$ and 0 otherwise;

- $\vec{x}^{s} \in\{0,1\}^{k}$ the vector representing its sign, such that: $\forall c \in \llbracket 1, k \rrbracket, \vec{x}^{s}(c)=0$ if $\vec{x}(c) \leq 0$ and $\vec{x}^{s}(c)=1$ otherwise.

Since the operators above are easily expressed using linear equations, we use them to reformulate the characterization proposition 4 . The new formulation will be used to build a linear programming model corresponding to the firing of a timed step sequence.

\subsubsection{Timed steps linear formulation}

In this section, we consider the equations (12) to (15) defining timed step firings and reformulate each of them using the discrimination operators given above. In order to avoid confusions of notations, we use lower case letters to denote the state vectors $\left(\overrightarrow{e_{m}}, \overrightarrow{e_{r}}\right)$ expressed using discrimination operators.

Proposition 7 (Timed step Firings Reformulation). Let $(R, d)$ be a Timed Petri net. Let $e=\left(\overrightarrow{e_{m}}, \overrightarrow{e_{r}}\right)$ be a state given at date $v$. Let $v^{\prime} \geq v$ and $\Delta_{v}=v^{\prime}-v \in \mathrm{N}$. Let $\psi^{\prime}=\left(\varphi^{\prime}, v^{\prime}\right)$ be a timed step. Then we have:

$$
\begin{aligned}
& e\left[\psi^{\prime}\right\rangle \Leftrightarrow\left\{\begin{array}{l}
\overrightarrow{\varphi^{\prime}} \leq \overrightarrow{1_{n}}-\left(\overrightarrow{e_{r}}-\Delta_{v} \cdot \overrightarrow{1_{n}}\right)^{s} \\
\overrightarrow{e_{m}}-C^{-} \cdot \overrightarrow{\varphi^{\prime}}+C^{+} \cdot\left(\overrightarrow{e_{r}}-\left(\overrightarrow{e_{r}}-\Delta_{v} \cdot \overrightarrow{1_{n}}\right)^{s}\right) \geq \overrightarrow{0_{m}}
\end{array}\right. \\
& \begin{array}{l}
e\left[\psi^{\prime}\right\rangle e^{\prime} \\
e^{\prime}=\left(\overrightarrow{e_{m}^{\prime}}, \overrightarrow{e_{r}^{\prime}}\right)
\end{array} \Leftrightarrow e\left[\psi^{\prime}\right\rangle \wedge\left\{\begin{array}{l}
\overrightarrow{e_{m}^{\prime}}=\overrightarrow{e_{m}}-C^{-} \cdot \overrightarrow{\varphi^{\prime}}+C^{+} \cdot\left(\overrightarrow{e_{r}}-\left(\overrightarrow{e_{r}}-\Delta_{v} \cdot \overrightarrow{1_{n}}\right)^{s}\right) \\
\overrightarrow{e_{r}^{\prime}}=\left(\overrightarrow{e_{r}}-\Delta_{v} \cdot \overrightarrow{1_{n}}\right)^{+}+\left.\sum_{t \in \mathrm{T}} d(t) \cdot \overrightarrow{\varphi^{\prime}}\right|_{t} \cdot \overrightarrow{e_{t}}
\end{array}\right.
\end{aligned}
$$

Proof. To prove the above proposition, one has just to verify that equations (21) to (24) correspond exactly to equations (12) to (15) from definition 19. 


\subsubsection{Illustrative example}

\begin{tabular}{|c|c|c|c|c|c|c|c|c|c|c|}
\hline \multirow[b]{2}{*}{ 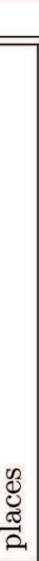 } & \multirow[b]{2}{*}{ 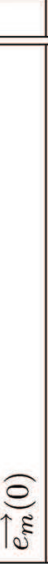 } & \multicolumn{3}{|c|}{$v_{0}=0, \Delta_{v_{0}}=1$} & \multicolumn{3}{|c|}{$v_{1}=1, \Delta_{v_{1}}=3$} & \multicolumn{3}{|c|}{$v_{2}=4, \Delta_{v_{2}}=2$} \\
\hline & & $\mid \begin{array}{c}0 \\
\vdots \\
4 \\
+ \\
\vdots \\
\vdots \\
0 \\
1 \\
0\end{array}$ & 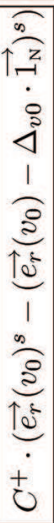 & 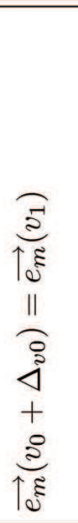 & 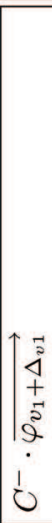 & 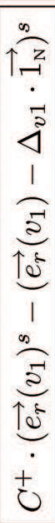 & 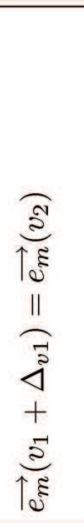 & 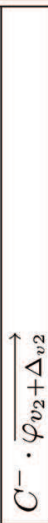 & 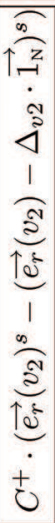 & 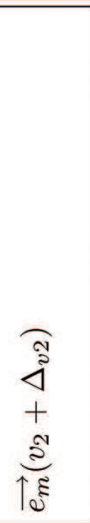 \\
\hline$p_{1}$ & 1 & 1 & 0 & 0 & 0 & 0 & 0 & 0 & 0 & 0 \\
\hline$p_{2}$ & 0 & 0 & 0 & 0 & 1 & 1 & 0 & 0 & 0 & 0 \\
\hline$p_{3}$ & 0 & 0 & 0 & 0 & 0 & 0 & 0 & 0 & 0 & 0 \\
\hline$p_{4}$ & 0 & 0 & 0 & 0 & 0 & 0 & 0 & 0 & 0 & 0 \\
\hline$p_{5}$ & 1 & 0 & 0 & 1 & 1 & 0 & 0 & 0 & 0 & 0 \\
\hline$p_{6}$ & 0 & 0 & 0 & 0 & 0 & 0 & 0 & 0 & 1 & 1 \\
\hline$r_{1}$ & 1 & 1 & 0 & 0 & 1 & 1 & 0 & 0 & 1 & 1 \\
\hline$r_{2}$ & 1 & 0 & 0 & 1 & 1 & 0 & 0 & 0 & 0 & 0 \\
\hline
\end{tabular}

Fig. 7. Intermediary Calculations (Markings)

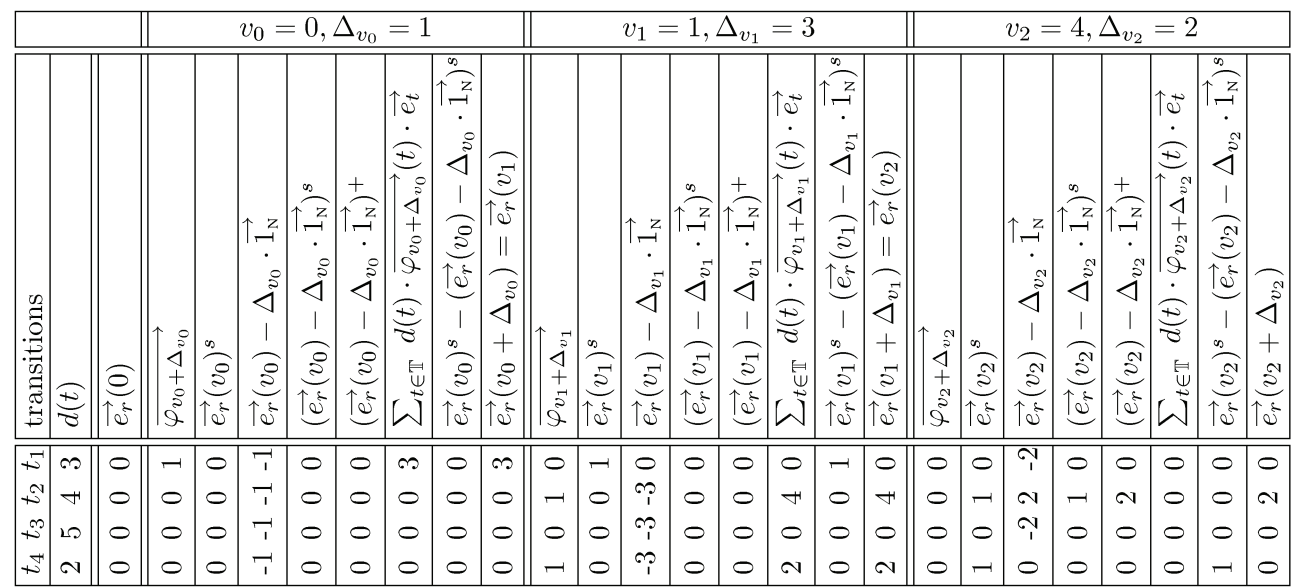

Fig. 8. Intermediary Calculations (Residual Durations)

In order to help understanding the above equations, we propose to illustrate them using a particular example. Let's consider the Timed Petri net of Fig. 3. Its initial marking at the date $v_{1}=0$ is given by: $\overrightarrow{e_{m}}(0)=(1,0,0,0,1,0,1,1)^{\text {ú }}, \overrightarrow{e_{r}}(0)=(0,0,0,0)^{\text {ú }}$. 
We assume that $t_{1}$ is fired at the date $v_{1}=1$, then simultaneously $t_{2}$ and $t_{4}$ at the date $v_{2}=4$. We need to calculate the state reached at the date $v_{3}=6$. The details of the calculation are given in Fig. 7 and 8. The physical sense of the equations is explained below:

- The quantity $\left(\overrightarrow{e_{r}}-\Delta_{v} \cdot \overrightarrow{1_{N}}\right)^{+}$represents the update of the residual durations vector at the date $v+\Delta_{v}$, from its value $\overrightarrow{e_{r}}$ at the date $v$. The " + " operator allows to take into account only positive values. Moreover, if a transition $t$ is still active at date $v+\Delta_{v}$, we have: $\left.\left(\overrightarrow{e_{r}}-\Delta_{v} \cdot \overrightarrow{1_{N}}\right)^{s}\right|_{t}=1$;

- The quantity $\left.\sum_{t \in \mathrm{T}} d(t) \cdot \overrightarrow{\varphi^{\prime}}\right|_{t} \cdot \overrightarrow{e_{t}}$ represents the new residual durations coming from the execution of the firing sequence $\varphi^{\prime}$ at the date $v+\Delta_{v}$;

- Finally, the quantity $\overrightarrow{e_{r}}-\left(\overrightarrow{e_{r}}-\Delta_{v} \cdot \overrightarrow{1_{N}}\right)^{s}$ represents the Parikh vector of the transitions, the firing of which ends at the date $v+\Delta_{v}$. This expression is made from the comparison between the Parikh vector of the transitions that were pending at the date $v$ : vector $\vec{e}_{r}^{s}$, and the Parikh vector of the transitions that will be still active at the date $v+\Delta_{v}$ : vector $\left(\overrightarrow{e_{r}}-\Delta_{v} \cdot \overrightarrow{1_{N}}\right)^{s}$.

\subsubsection{Mathematical programming model}

Since proposition 7 has been formulated in a linear way, it allows to express the linear mathematical programming model given below.

Model 2 (TPN Integer Programming Model). Let $(R, d)$ be a TPN with its initial state $e_{0}=\left(\overrightarrow{e_{m_{0}}}, \overrightarrow{0_{N}}\right)$ given at date $v_{0}=0$. Let $e_{f}=\left(\overrightarrow{e_{m_{f}}}, \overrightarrow{0_{N}}\right)$ be a target state. Let $V \in \mathrm{N}$.

The integer linear programming model $\mathrm{TIP}(V)$ is defined by:

$$
\text { Minimize } \sum_{i \in \llbracket 0, V-1 \rrbracket} \Delta_{v_{i}}
$$

subject to:

$$
\begin{aligned}
& \forall k \in \llbracket 1, M \rrbracket, \quad \quad e_{m 0 k} \quad=\left.\overrightarrow{e_{m_{0}}}\right|_{k} \\
& \forall k \in \llbracket 1, M \rrbracket, \quad e_{m V k} \quad=0
\end{aligned}
$$




$\begin{array}{llll}\forall j \in \llbracket 1, N \rrbracket, & e_{r 0 j} & = & \overrightarrow{e_{r_{0}}} \\ \forall j \in \llbracket 1, N \rrbracket, & e_{r V j} & = & 0\end{array}$

$\forall i \in \llbracket 1, V \rrbracket, \quad \forall j \in \llbracket 1, N \rrbracket$,

$$
B \cdot a_{i j}-e_{r i j} \quad \leq B-1
$$

$\forall i \in \llbracket 1, V \rrbracket, \quad \forall j \in \llbracket 1, N \rrbracket$,

$$
e_{r i j}-B \cdot a_{i j} \leq 0
$$

$\forall i \in \llbracket 1, V \rrbracket, \quad \forall j \in \llbracket 1, N \rrbracket$,

$$
B \cdot \alpha_{i j}-e_{r i j}+\Delta_{v_{i}} \leq B-1
$$

$\forall i \in \llbracket 1, V \rrbracket, \quad \forall j \in \llbracket 1, N \rrbracket$,

$$
e_{r i j}-\Delta_{v_{i}}-B \cdot \alpha_{i j} \leq 0
$$

$\forall i \in \llbracket 1, V \rrbracket, \quad \forall j \in \llbracket 1, N \rrbracket$,

$$
e_{r i j}-\Delta_{v_{i}}-\beta_{i j} \leq 0
$$

$\forall i \in \llbracket 1, V \rrbracket, \quad \forall j \in \llbracket 1, N \rrbracket$,

$$
\beta_{i j}-B \cdot \alpha_{i j} \leq 0
$$

$\forall i \in \llbracket 1, V \rrbracket, \quad \forall j \in \llbracket 1, N \rrbracket, \quad \beta_{i j}+B \cdot \alpha_{i j}-e_{r i j}+\Delta_{v_{i}} \leq \quad B$

$\forall i \in \llbracket 1, V \rrbracket, \quad \forall k \in \llbracket 1, M \rrbracket, \quad e_{m i k}-e_{m(i-1) k}+\sum_{c=1}^{N} C_{k c}^{-} \cdot \varphi_{i c}$

$$
-\sum_{c=1}^{N} C_{k c}^{+} \cdot\left(a_{(i-1) c}-\alpha_{(i-1) c}\right)=0
$$

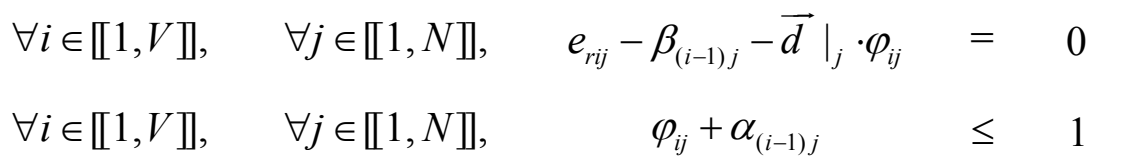

$\forall i \in \llbracket 1, V \rrbracket, \quad \forall j \in \llbracket 1, N \rrbracket$,

$\begin{array}{ll}\varphi_{i j} & \in\{0,1\} \\ a_{i j} & \in\{0,1\} \\ \alpha_{i j} & \in\{0,1\} \\ \beta_{i j} & \in \mathrm{N}\end{array}$

$\forall i \in \llbracket 1, V \rrbracket, \quad \forall j \in \llbracket 1, N \rrbracket$,

$\forall i \in \llbracket 1, V \rrbracket, \quad \forall j \in \llbracket 1, N \rrbracket$,

$\forall i \in \llbracket 1, V \rrbracket, \quad \forall j \in \llbracket 1, N \rrbracket$,

$e_{r i j} \quad \in \quad \mathrm{N}$

$\forall i \in \llbracket 1, V \rrbracket, \quad \forall j \in \llbracket 1, N \rrbracket$, 


$$
\begin{array}{ccccc}
\forall i \in \llbracket 1, V \rrbracket, & \forall k \in \llbracket 1, M \rrbracket, & e_{\text {mik }} & \in & \mathrm{N} \\
\forall i \in \llbracket 0, V-1 \rrbracket, & \Delta_{v_{i}} & \in & \mathrm{N}
\end{array}
$$

Equations (26) to (29) correspond to conditions over initial and final states. Equations (30) to (36) express the constraints over discrimination variables used to compute the " + " and " $s$ " operators. Variables $\left(a_{i}\right),\left(\alpha_{i}\right)$ and $\left(\beta_{i}\right)$ denote respectively the values of $\overrightarrow{e_{r}}\left(v_{i}\right)^{s}$, $\left(\overrightarrow{e_{r}}\left(v_{i}\right)-\Delta_{v_{i}} \cdot \overrightarrow{1_{n}}\right)^{s}$ and $\left(\overrightarrow{e_{r}}\left(v_{i}\right)-\Delta_{v_{i}} \cdot \overrightarrow{1_{n}}\right)^{+}$from equations (23) and (24). Equations (37) and (38) correspond to intermediate state computation equations (23) and (24). Equation (39) correspond to nonreentrance condition (21). Finally, equations (40) to (46) define the domain of the used variables.

Again, our model is well defined enough to allow the following proposition.

Proposition 8 (Correctness and completeness of $\operatorname{TIP}(\mathbf{v})$ w.r.t. $\left.\operatorname{TP}_{\mathbf{1}}(\mathbf{v})\right)$. Let $(R, d)$ be a TPN and $v \in \mathrm{N}$. Then we have:

- Any solution of $\mathrm{TIP}(v)$ is also a solution of $\mathrm{TP}_{1}(v)$ (Correctness)

- Any solution of $\mathrm{TP}_{1}(v)$ can be expressed as a solution of $\mathrm{TIP}(v)$ (Completeness)

Proof. Those results come directly from the construction of TIP(V).

Obviously, the same remarks as for proposition 5 hold. Even if problem $\mathrm{TP}_{1}$ is parametrized by a given number of timed steps, a large class of scheduling problems can be adressed using such formulation. We are more particularly interested in flexible manufacturing systems (FMS) scheduling problems. FMS are characterized by the simultaneous production of several types of products, and the possibility to use several methods (flexibilities) to produce the same kind of product. Using TPN, such flexibilities are modeled by conflicts, which justifies the use of our approach.

Another interest in the framework of FMS is the formulation of cyclic scheduling problems in a smart way. Indeed: such scheduling problems correspond to reachability between the same states (Bourdeaud'huy and Korbaa, 2006). Using our approach, one can formulate a cyclic scheduling problem by considering a timed reachability problem between two identical unknown states. The corresponding model allows then not only to find the schedule but also the initial state within the cycle.

Note finally that our mathematical model remains valid to solve the reachability problem between states defined by not null residual durations. One has just to consider that these states belong to a bigger problem between states without residual durations.

\subsubsection{Numerical experiments}

In order to validate the model above, preliminary experiments were carried out using the linear programming solver CPLEX 9.0. (Bourdeau'huy et al., 2006). They have shown promising results, but need to be extended in order to assess the efficiency of our approach compared to concurrent approaches from Operations Research litterature. 
We also propose to develop cutting techniques allowing to improve the resolution performances. For instance, we suggest to reuse the decomposition technique described above. A preliminary resolution of the reachability problem between the initial and final state vectors in the underlying $\mathrm{P} / \mathrm{T}$ Petri net can be used to obtain the Parikh vector of the firing sequence of the controlled execution searched for.

\section{Conclusion and future work}

In this chapter, we present techniques for solving reachability problems in PN and TPN based on mathematical programming. The approach is based on an incremental search using step sequences that represent parallel and reentrant firings of transitions. The mathematical model used allows the formulation and verification of reachability-based analysis problems.

Concerning PNs, we have proposed two formulations of the reachability problem, leading to integer and/or binary programming models. For each of them, we have developped some additional procedures, relaxation techniques and objective functions in order to improve the computational efficiency of the resolution. Numerical experiments have demonstrated the efficiency of our approach compared to standard ones from Artificial Intelligence and Petri nets community.

Several promising tracks will be considered in the future, such that:

- To develop rules to adjust dynamically the amplitudes of jump search, for example by exploiting information from the previous iterations and/or from the structure of the considered PN;

- $\quad$ To use heuristic methods to speed up the search or find a good bound on $K_{\text {min }}$.

Concerning TPN, we have shown how a linear integer programming model could be developped to solve the Timed Petri net reachability problem. This model is very general since it allows to deal with weighted Timed Petri nets, without restricting ourselves to an immediate firing semantic or Timed Event Graphs as it is done in the litterature. It can thus be directly used on flexible manufacturing models.

In the future, we propose to compare our computational results with concurrent approaches dedicated to scheduling problems. We also propose to develop cutting techniques allowing to improve the resolution performances.

Finally, we are currently adapting our incremental approaches to Time Petri nets, in order to be able to model scheduling problems with Time Windows associated to the tasks.

\section{References}

Baccelli, F., Cohen, G., Olsder, G., and Quadrat, J.-P. (1992). Synchronization and linearity:

An algebra for Discrete Event Systems. Wiley, New York

Benasser, A. (2000). L'accessibilité dans les réseaux de Petri : une approche basée sur la programmation par contraintes. PhD thesis, Université des sciences et techologies de Lille

Benasser, A. and Yim, P. (1999). Railway Traffic Planning with Petri nets and Constraint Programming. JESA, 33(8-9), pp. 959-975

Berthelot, G. (1986). Transformations and Decompositions of Nets. Advances in Petri Nets 1986 Part I, Proceedings of an Advanced Course, Vol. 254, pp. 359-376 
Berthomieu, B. and Diaz, M. (1991). Modeling and Verification of Time Dependent Systems using Time Petri Nets. IEEE Trans. on Software Eng., 17(3), pp. 259-273

Bourdeaud'huy, T. (2004). Techniques d'Abstraction pour l'Analyse et la Synthèse de Réseaux de Petri. PhD thesis, Ecole Centrale de Lille

Bourdeaud'huy, T., Hanafi, S., and Yim, P. (2004a). Efficient Reachability Analysis of Bounded Petri nets using Constraint Programming. SMC'04, International Conference on Systems, Man and Cybernetics, La Hague, Hollande

Bourdeaud'huy, T., Hanafi, S., and Yim, P. (2004b). Recherche de Séquences d'Accessibilité dans les Réseaux de Petri utilisant l'Abstraction Logique et une réduction fondée sur l'équation d'état. CIFA'04, Conférence Internationale Francophone d'Automatique, Douz, Tunisie

Bourdeaud'huy, T., Hanafi, S., and Yim, P. (2004c). Solving the Petri Nets Reachability Problem using the Logical Abstraction Technique and Mathematical Programming. CPAIOR'04, International Conference on Integration of AI and OR Techniques in Constraint Programming for Combinatorial Optimisation Problems, LNCS 3011, pp. 112-127, Nice, France

Bourdeaud'huy, T., Hanafi, S., and Yim, P. (2006). Scheduling of Flexible Manufacturing Systems using Timed Petri nets and Mathematical Programming. WODES'06, Workshop on Discrete Event Systems, Ann Arbor, Michigan, USA

Bourdeaud'huy, T., Hanafi, S., and Yim, P. (2007). Mathematical Programming Approach for the Petri Nets Reachability Problem. EJOR, 177(1)

Bourdeaud'huy, T. and Korbaa, O. (2006). A Mathematical Model for Cyclic Scheduling with Work-In-Progress Minimization. INCOM'06, IFAC Symposium on Information Control Problems in Manufacturing, Saint Etienne, France

Briand, C. (1999). Solving the Car-Sequencing Problem using Petri Nets. International Conference on Industrial Engineering and Production Management, Vol. 1, pp. 543551

Chrétienne, P. (1984). Exécutions Contrôlées dans les Réseaux de Petri Temporisés. T.S.I., 3

Colom, J. and Silva, M. (1989a). Convex Geometry and Semiflows in P/T nets: a Comparative Study of Algorithms for Computation of Minimal P-semiflows. Proceedings of the 10th International Conference on Application and Theory of Petri Nets

Colom, J. and Silva, M. (1989b). Improving the Linearly based Characterization of P/T nets. Proceedings of the 10th International Conference on Application and Theory of Petri nets, pp. 52-73, Bonn, Germany

David, R. and Alla, H. (1992). Petri Nets and Grafcet: Tools for Modelling Discrete Event Systems. Prentice-Hall

Fernandez, J.-C., Jard, C., Jéron, T., andMounier, L. (1992). “'On the fly” Verification of Finite Transition Systems. Formal Methods in System Design

Geoffrion, A. (1974). Lagrangean Relaxation for Integer Programming. Mathematical Programming Study, Vol. 2, pp. 82-114

Glover, F. (1977). Heuristics for Integer Programming using Surrogate Constraints. Decision Sciences, Vol. 8, pp. 156-166 
Gunnarsson, J. (1998). Symbolic Tools for Verification of Large Scale DEDS. Proc. IEEE Int. Conf. on Systems, Man, and Cybernetics (SMC'98), 11-14 October 1998, San Diego, CA, pp. 722-727.

Hillion, H. and Proth, J. (1989). Performance Evaluation of Job-Shop Systems using Timed Event Graphs. IEEE Transactions on Automatic Control, Vol. 34

Huber, P., Jensen, A. M., Jepsen, L. O., and Jensen, K. (1985). Towards Reachability Trees for High-level Petri Nets. Lecture Notes in Computer Science: Advances in Petri Nets 1984, Vol. 188, pp. 215-233

Jaffar, J., Michaylov, Stuckey, P., and Yap, R. (1992). The CLP (R) Language and System. ACM Transactions on Programming Languages and Systems, Vol. 14(3), pp. 339395

Janicky, R. and Koutny, M. (1991). Optimal Simulations, nets and Reachability Graphs. Advances in Petri Nets, Lecture Notes In Computer Science, Vol. 524, pp. 205-226

Jensen, K. (1992). Coloured Petri nets - Basic Concepts, Analysis Methods and Practical Use. EATCS Monographs on Theoretical Computer Science, Vol. 1, pp. 1-234. Springer

Keller, R. (1976). Formal Verification of Parallel Programs. Comm. of the ACM, Vol. 19(7), pp. 371-384

Khomenko, V. and Koutny, M. (2000). Verification of Bounded Petri Nets using Integer Programming, technical report cs-tr-711, Department of Computing Science, University of Newcastle upon Tyne

Kosaraju, S. R. (1982). Decidability of Reachability in Vector Addition Systems. Proc. Of the 14th Annual ACM Symp. on Theory of Computing, pp. 267-281

Latvala, T. (2001). Model checking LTL Properties of High-level Petri Nets with Fairness Constraints. Lecture Notes in Computer Science 2075

Lautenbach, K. (1987). Linear Algebraic Techniques for P/T Nets. Advances in Petri Nets 1986, Part I, Proceedings of an Advanced Course, Vol. 254, pp. 142-167

Lee, D. Y. and DiCesare, F. (1994). Scheduling Flexible Manufacturing Systems using Petri nets and Heuristic Search. IEEE Transactions on Robotics and Automation, Vol. 10(2), pp. 123-132

Lindqvist, M. (1993). Parameterized Reachability Trees for Predicate/Transition Nets. Lecture Notes in Computer Science; Advances in Petri Nets 1993, Vol. 674, pp. 301324

Lipton, R. (1976). The Reachability Problem requires Exponential Space. Technical report, Computer Science Dept., Yale University

Melzer, S. and Esparza, J. (1996). Checking System Properties via Integer Programming. ESOP'96

Murata, T. (1989). Petri Nets : Properties, Analysis and Applications. Proceedings of the IEEE, Vol. 77, pp. 541-580

Parker, R. and Rardin, R. (1988). Discrete Optimization. Academic Press

Ramchandani, C. (1974). Analysis of Asynchronous Concurrent Systems by Timed Petri Nets. PhD thesis, Massachusetts Institute of Technology

Richard, P. (2000). Modelling Integer Linear Programs with Petri nets. RAIRO/Operations Research, Vol. 34(3), pp. 305-312

Roch, S. and Starke, P. (2002). INA Manual, Integrated Net Analyzer, Version 2.2. Humboldt- Universität zu Berlin, Institut für Informatik 
Schmidt, K. (1998). On the new Low Level Symmetry tool in INA. GI Petri Net Newsletter 54

Sifakis, J. (1975). Performance Evaluation of Systems using Nets. Advanced Course: Net Theory and Applications, pp. 307-319

Silva, F., Castilho, M. A., and Kunzle, L. A. (2000). Petriplan: A new Algorithm for Plan Generation (preliminary report). IBERAMIA-SBIA, pp. 86-95

Silva, M., Colom, J., and Campos, J. (1992). Linear Algebraic Techniques for the Analysis of Petri Nets. Recent Advances in Mathematical Theory of Systems, Control, Networks, and Signal Processing II

Silva,M., Teruel, E., and Colom, J.M. (1998). Linear Algebraic and Linear Programming Techniques for the Analysis of P/T Net Systems. Lecture Notes in Computer Science: Lectures on Petri Nets I: Basic Models, Vol. 1491, pp. 309-373

Valmari, A. (1991). Stubborn Sets for Reduced State Space Generation. Lecture Notes in Computer Science; Advances in Petri Nets 1990, Vol. 483, pp. 491-515

Van der Aalst,W.M. P. (1995). Petri Net Based Scheduling. Number 95. Eindhoven University of Technology Computing Science Reports/23

Vernadat, F., Azéma, P., and Michel, P. (1996). Covering Steps Graphs. 17th Int. Conf on Application and Theory of Petri Nets 96.

Wang, J. (1998). Timed Petri Nets, Theory and Application. Kluwer Academic Publishers 


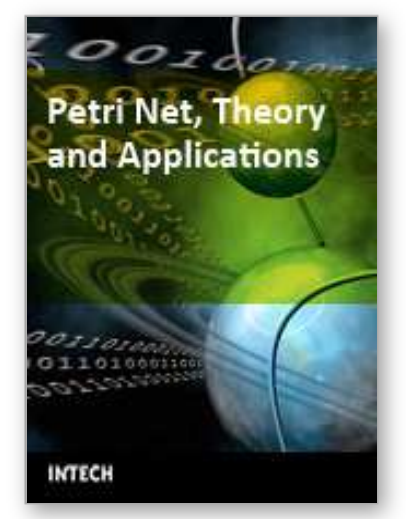

\author{
Petri Net, Theory and Applications \\ Edited by Vedran Kordic
}

ISBN 978-3-902613-12-7

Hard cover, 534 pages

Publisher I-Tech Education and Publishing

Published online 01, February, 2008

Published in print edition February, 2008

Although many other models of concurrent and distributed systems have been de- veloped since the introduction in 1964 Petri nets are still an essential model for concurrent systems with respect to both the theory and the applications. The main attraction of Petri nets is the way in which the basic aspects of concurrent systems are captured both conceptually and mathematically. The intuitively appealing graphical notation makes Petri nets the model of choice in many applications. The natural way in which Petri nets allow one to formally capture many of the basic notions and issues of concurrent systems has contributed greatly to the development of a rich theory of concurrent systems based on Petri nets. This book brings together reputable researchers from all over the world in order to provide a comprehensive coverage of advanced and modern topics not yet reflected by other books. The book consists of 23 chapters written by 53 authors from 12 different countries.

\title{
How to reference
}

In order to correctly reference this scholarly work, feel free to copy and paste the following:

Thomas Bourdeaud'huy, Said Hanafi and Pascal Yim (2008). Incremental Integer Linear Programming Models for Petri Nets Reachability Problems, Petri Net, Theory and Applications, Vedran Kordic (Ed.), ISBN: 978-3902613-12-7, InTech, Available from:

http://www.intechopen.com/books/petri_net_theory_and_applications/incremental_integer_linear_programmin g_models_for_petri_nets_reachability_problems

\section{INTECH}

open science | open minds

\section{InTech Europe}

University Campus STeP Ri

Slavka Krautzeka 83/A

51000 Rijeka, Croatia

Phone: +385 (51) 770447

Fax: +385 (51) 686166

www.intechopen.com

\section{InTech China}

Unit 405, Office Block, Hotel Equatorial Shanghai

No.65, Yan An Road (West), Shanghai, 200040, China

中国上海市延安西路65号上海国际贵都大饭店办公楼 405 单元

Phone: +86-21-62489820

Fax: $+86-21-62489821$ 
(C) 2008 The Author(s). Licensee IntechOpen. This chapter is distributed under the terms of the Creative Commons Attribution-NonCommercialShareAlike-3.0 License, which permits use, distribution and reproduction for non-commercial purposes, provided the original is properly cited and derivative works building on this content are distributed under the same license. 